\title{
A Family of Non Linear Models in a Market with Semi Markov Regimes: Application to the Commodity and the Derivative Market
}

\author{
Patrick Assonken ${ }^{1} \&$ Gangaram S. Ladde ${ }^{2}$ \\ ${ }^{1}$ Department of Mathematics, Coastal Pines Technical College, Brunswick, Georgia, USA \\ 2 Department of Mathematics and Statistics, University of South Florida, Tampa, Florida USA \\ Correspondence: Patrick Assonken, 820 Scranton Rd. Apt\#303, Brunswick, Georgia 31525 USA. \\ Email: passonken@coastalpines.edu
}

Received: August 18, 2017 Accepted: September 1, 2017 Online Published: October 20, 2017

doi:10.5539/ijsp.v6n6p137

URL: https://doi.org/10.5539/ijsp.v6n6p137

\begin{abstract}
This paper introduces a family of coupled semi Markov regime switching multidimensional non linear models for general asset prices. Two particular instances of the models are explored. The first instance is one modeling commodity prices. Estimation formulas for historical parameters are developed. The second instance of the family of models introduced is one generalizing Heston model. It allows for semi Markov regime switching of Heston parameters. We develop a general semi closed formula for vanilla option prices given the risk neutral option parameters.
\end{abstract}

Keywords: LLGMM, Semi Markov Process, Regime Switching, Heston Model, Commodity Market, Option pricing

\section{Introduction}

We introduce a family of coupled semi Markov regime switching multidimensional non linear models extending the commodity model developed by Otunuga and Ladde (2014); Otunuga et al. (2016), most stochastic volatility models and local volatility models, namely, Heston-type models discussed by Heston (1993); Da Fonseca et al. (2008); Papanicolaou and Sircar (2014), Constant Elasticity of Volatility (CEV) type models, Garch models and Cox-Ingerson-Ross (CIR) models discussed by Geman and Shih (2009); Cox et al. (1985); Duan et al. (1995) among others. Stochastic volatility models are ubiquitous in financial modeling, as they are a significant upgrade over Black Scholes model introduces by Black and Scholes (1973) both in derivative pricing and in asset return prediction. In derivative pricing, stochastic volatility model provide an implied volatility surface in compliance with many empirical features of the market such as smiles and smirks. As for historical parameter estimation, Stochastic volatility models provide a log return distribution exhibiting many empirically observed features (skewness, fatness of tails and high peak). Despite the abundant literature of Heston models and its well documented reproduction of many stylized empirical facts, a semi Markov switching market has the potential of adding to the already well documented flexibility of the model. Semi Markov regime switching market represent a non obvious generalization of the more common Markov regime switching models. Such models have been studied recently by Assonken and G. Ladde $(2016,2015)$ who finds a Fourier methods through a characteristic function formula, for pricing derivatives in Levy driven financial markets.

The families of models introduced also allow the modeling of multi-asset baskets. Financial portfolios are often divided in sectors (technology, energy, commodity and so on) which are assumed to show significant intra-sector correlation and little to no inter-sector correlation. Hence prediction of one specific asset price could be improved when accounting for prices of assets in the same group. Otunuga and Ladde (2014); Otunuga et al. (2016) first developed a calibration technique for such a class of model, namely the LLGMM method, accounting for a unique layer of interaction between asset prices through their diffusion coefficients. We extend such a model to semi Markov markets and provide in the same token an opportunity to assess the unpredictable jumps effects on the calibration results. The rest of the chapter is organized as follows: Section 2 is an introduction to the general multidimensional non linear model. Section 3 is concerned with the two main applications: the special case of a coupled multidimensional stochastic differential equation model describing commodity prices and an extension of Heston model to account for semi Markov regime changes and a regime switching Heston Model.

\section{Preliminary Notation and Results}

Let $(\Omega, \mathbb{F})$ and $m$ be the reference measurable space and a whole number, respectively. We denote $T^{*}, T$ and $\psi$ the market time horizon and the maturity time of some derivative contract, and a Poisson random measure, respectively. $\left(\theta_{t}\right)_{t \in\left[0, T^{*}\right]}$ is a semi Markov process with state space $E=\{1,2, \ldots, m\}$, state switching times $\left(T_{k}\right)_{k \in \mathbb{N}}$ and $\left(\boldsymbol{\beta}_{k}\right)_{k \in \mathbb{N}}$ is a discrete sequence of random non negative vector valued numbers with $\boldsymbol{\beta}_{k}=\left(\beta_{k}^{1}, \beta_{k}^{2}, \ldots, \beta_{k}^{n}\right)$ and $\ln \left(\boldsymbol{\beta}_{k}\right) \sim \overline{\boldsymbol{b}}$, where $\overline{\boldsymbol{b}}$ is a $n$-dimensional 
probability density function. We will also use the notation $\theta_{T_{k}}=\theta_{k}$ for simplicity. We consider $n, q, l \in \mathbb{N}^{\star}$ and $\boldsymbol{x}$ an $n$-dimensional vector stochastic process, $\boldsymbol{\mu} \in C\left[\mathbb{R}_{+} \times \mathbb{R}_{+} \times E \times \mathbb{R}^{n}, \mathbb{R}^{n}\right], \boldsymbol{\sigma} \in C\left[\mathbb{R}_{+} \times \mathbb{R}_{+} \times E \times \mathbb{R}^{n}, \mathbb{R}^{n} \times \mathbb{R}^{l}\right], \boldsymbol{B}$ is a $l$-dimensional vector of independent Brownian motions, $\boldsymbol{G}, \mathbf{H} \in C\left[\mathbb{R}_{+} \times \mathbb{R}_{+} \times E \times \mathbb{R}^{n} \times \mathbb{R}, \mathbb{R}^{n}\right]$ and $R \in \mathcal{B}\left[\mathbb{R} \times \mathbb{R} \times \mathbb{R}^{n} \times \mathbb{R}^{n}, \mathbb{R}^{n}\right]$ a bounded function.

$\psi(j),, v(j$,$) and L_{j}$ are respectively a Poisson process, its intensity measure, and the cadlág Levy processes with characteristic triplets $((\mu)(j),, \sigma(j),, v(j)$,$) when the semi Markov process \theta$ is in the $j$-th state. We assume that the following inequality holds:

$$
\int_{0}^{T} \int_{z \in \mathbb{R}}\left(\left(1+\boldsymbol{H}^{T}\left(t, y_{t}, j, x_{t}, z\right) \boldsymbol{H}\left(t, y_{t}, j, x_{t}, z\right)\right) 1_{|z|>1}+\boldsymbol{G}^{T}\left(t, y_{t}, j, x_{t}, z\right) \boldsymbol{G}\left(t, y_{t}, j, x_{t}, z\right) 1_{|z| \leq 1}\right) v(j, d z)<\infty, \forall j \in E
$$

$. \boldsymbol{\mu}, \boldsymbol{\sigma}, \boldsymbol{G}$ and $\boldsymbol{H}$ are assumed smooth enough to ensure existence of a solution of the following system of stochastic partial differential equations:

$$
\begin{aligned}
& d \boldsymbol{x}_{t}=\boldsymbol{\mu}\left(t, y_{t^{-}}, \theta_{k}, \mathbf{x}_{t^{-}}\right) d t+\boldsymbol{\sigma}\left(t, y_{t^{-}}, \theta_{k}, \boldsymbol{x}_{t^{-}}\right) d \mathbf{B}_{t}+\int_{|z|<1} \boldsymbol{G}\left(t, y_{t^{-}}, \theta_{k}, \boldsymbol{x}_{t^{-}}, z\right) \bar{\psi}\left(\theta_{k}, d t, d z\right) \\
& +\int_{|z|>1} \boldsymbol{H}\left(t, y_{t^{-}}, \theta_{k}, \boldsymbol{x}_{t}, z\right) \psi\left(\theta_{k}, d t, d z\right) \\
& \boldsymbol{x}_{k}=\boldsymbol{\beta}_{k} \boldsymbol{x}_{k}^{-}, \forall k \in I(1, \infty)=\mathbb{N} \text {, where } \boldsymbol{x}_{T_{k}}=\boldsymbol{x}_{k}, \forall k \in I(1, \infty)=\mathbb{N}, \forall t \in\left[T_{k}, T_{k+1}\right), \ln \left(\boldsymbol{\beta}_{k}\right) \sim \overline{\boldsymbol{b}}\left(\mid \theta_{k-1}, \theta_{k}\right)
\end{aligned}
$$

$\left(\mathbb{H}_{t}\right)_{t \in[0, T]},\left(\mathbb{L}_{t}\right)_{t \in[0, T]}$ and $\mathbb{B}_{n}$ are filtration generated by the semi Markov process $\theta_{t}$, Levy processes $L_{s}^{j}, s \in[0, t], \forall j \in$ $E=\{1,2,3, \ldots, m\}$ and the discrete vector sequence $\left(\boldsymbol{\beta}_{k}\right)_{k \in \mathbb{N}}$, respectively. We also denote $\overline{\mathbb{L}}_{t}=\mathbb{L}_{t} \vee \mathbb{B}_{n(t)}, \overline{\mathbb{G}}_{t}=\mathbb{H}_{T} \vee \overline{\mathbb{L}}_{t}$ and $\mathbb{G}_{t}=\mathbb{H}_{t} \vee \overline{\mathbb{L}}_{t}, \forall t \in\left[0, T^{\star}\right]$. Let $P$ and $Q$ be the historical probability and an equivalent martingale measures as specified by Assonken and G. Ladde (2015), respectively, associated with the price process $(\boldsymbol{x}(t))_{t>0}$ defined on the reference space $(\Omega, \mathbb{F})$. We present an extension of Itó's lemma developed by Assonken and G. Ladde (2015), for a function $V \in C^{1,1,0,2}\left[\mathbb{R}^{+} \times \mathbb{R}^{+} \times E \times \mathbb{R}^{n}, \mathbb{R}^{q}\right]$. $\frac{\partial V}{\partial x}$ denotes the $q \times n$ first derivative matrix of $V$ and $\frac{\partial^{2} V}{\partial x^{2}}=\left(\frac{\partial^{2} V_{k}}{\partial x_{i} \partial x_{j}}\right)_{n \times n \times q}$ represents a tensor of rank 3. We use the following notation: $\boldsymbol{G}=\left(G_{1}, \ldots, G_{q}\right)^{T}, \boldsymbol{H}=\left(H_{1}, \ldots, H_{q}\right)^{T}$ and $\operatorname{Tr}\left[a^{T} \frac{\partial^{2} V}{\partial x^{2}} a\right]=$ $\left(\operatorname{Tr}\left(a^{T} \frac{\partial^{2} V_{1}}{\partial x^{2}} a\right), \ldots, \operatorname{Tr}\left(a^{T} \frac{\partial^{2} V_{q}}{\partial x^{2}} a\right)\right)^{T}, \forall a \in \mathbb{R}^{n} \times \mathbb{R}^{p}, \forall p \in \mathbb{N}^{*}$. We define the following set $E^{\star}=E^{2}-\{(i, i), i \in E\}$. We denote $N(t, A, B)$ a marked point process on $[0, T] \times \mathbb{B}(\mathbb{R}) \times P\left(E^{\star}\right)$ into $[0, \infty)$ as follows:

$$
N(t, A, B)=\sum_{n \geq 1} 1_{\left(t \geq T_{n}, \ln \left(\beta_{n}\right) \in A,\left(\theta_{n-1}, \theta_{n}\right) \in B\right)}
$$

$N(t, A, B)$ is the number of regime switches in subset $B$ of $E^{\star}$ with corresponding $\log$ price jumps $\ln \left(\beta_{n}\right) \in A \subset \mathbb{R}$ by time $t$. The compensator process

$$
\gamma(t, A, B)=\sum_{(i, j) \in B} \int_{0}^{t} \int_{z \in A} \overline{\boldsymbol{b}}(z \mid i, j) \lambda_{i, j}\left(y_{s}\right) d z d s
$$

of $N(t, A, B)$ and Itó formula for a suitable function $V$ are derived by Assonken and G. Ladde (2015). A multidimensional 
version of the formula can be summarized as follows:

$$
\begin{aligned}
& d V\left(s, y_{s}, \theta_{s}, \boldsymbol{x}_{s}\right)=\frac{\partial V}{\partial t} d t+\frac{\partial V}{\partial y} d y+\frac{\partial V}{\partial \boldsymbol{x}} d \boldsymbol{x}_{s}+\frac{1}{2}\left(d \boldsymbol{x}_{s}^{c}\right)^{T} \frac{\partial^{2} V}{\partial \boldsymbol{x}^{2}} d \boldsymbol{x}_{s}^{c} \\
& +\int_{|z| \leq 1}\left[V\left(s, y_{s^{-}}, \theta_{s^{-}}, \boldsymbol{x}_{s^{-}}+\boldsymbol{G}\left(s, y_{s^{-}}, \theta_{s^{-}}, \boldsymbol{x}_{s^{-}}, z\right)\right)-V\left(s, y_{s^{-}}, \theta_{s^{-}}, \boldsymbol{x}_{s^{-}}\right)\right. \\
& \left.-\frac{\partial V}{\partial \boldsymbol{x}} \boldsymbol{G}\left(s, y_{s^{-}}, \theta_{s^{-}}, \boldsymbol{x}_{s^{-}}, z\right)\right] \bar{\psi}\left(\theta_{s^{-}}, d z, d s\right) \\
& +\int_{|z|>1}\left[V\left(s, y_{s^{-}}, \theta_{s^{-}}, \boldsymbol{x}_{s^{-}}+\boldsymbol{H}\left(s, y_{s^{-}}, \theta_{s^{-}}, \boldsymbol{x}_{s^{-}}, z\right)\right)-V\left(s, y_{s^{-}}, \theta_{s^{-}}, \boldsymbol{x}_{s^{-}}\right)\right. \\
& \left.-\frac{\partial V}{\partial \boldsymbol{x}} \boldsymbol{H}\left(s, y_{s^{-}}, \theta_{s^{-}}, \boldsymbol{x}_{s^{-}}, z\right)\right] \psi\left(\theta_{s^{-}}, d z, d s\right) \\
& \int_{|z| \leq 1}\left[V\left(s, y_{s^{-}}, \theta_{s^{-}}, \boldsymbol{x}_{s^{-}}+\boldsymbol{G}\left(s, y_{s^{-}}, \theta_{s^{-}}, \boldsymbol{x}_{s^{-}}, z\right)\right)-V\left(s, y_{s^{-}}, \theta_{s^{-}}, \boldsymbol{x}_{s^{-}}\right)\right. \\
& \left.-\frac{\partial V}{\partial \boldsymbol{x}} \boldsymbol{G}\left(s, y_{s^{-}}, \theta_{s^{-}}, \boldsymbol{x}_{s^{-}}, z\right)\right] v\left(\theta_{s^{-}}, d z\right) d s \\
& +\sum_{j=1, j \neq \theta_{s^{-}}}^{m} \int_{z \in \mathbb{R}}\left[V\left(s, y_{s}, j, \boldsymbol{x}_{s^{-}} e^{z}\right)-V\left(s, y_{s^{-}}, \theta_{s^{-}}, \boldsymbol{x}_{s^{-}}\right)\right] N\left(d z, d s,\left\{\left(\theta_{s^{-}}, j\right)\right\}\right) \\
& =\frac{\partial V}{\partial s} d s+\frac{\partial V}{\partial y} d y+\frac{\partial V}{\partial \boldsymbol{x}} \boldsymbol{\mu}\left(t, \theta_{k}, y_{s^{-}}, \boldsymbol{x}_{s^{-}}\right) d s+\operatorname{Tr}\left[\frac{1}{2} \boldsymbol{\sigma}^{T}\left(s, y_{s^{-}}, \theta_{s^{-}}, \boldsymbol{x}_{s^{-}}\right) \frac{\partial^{2} V}{\partial \boldsymbol{x}^{2}} \boldsymbol{\sigma}\left(s, y_{s^{-}}, \theta_{s^{-}}, \boldsymbol{x}_{s^{-}}\right)\right] d s \\
& +\frac{\partial V}{\partial \boldsymbol{x}} \boldsymbol{\sigma}\left(s, \theta_{k}, y_{s^{-}}, \boldsymbol{x}_{s^{-}}\right) d \boldsymbol{B}_{s} \\
& +\int_{|z| \leq 1}\left[V\left(s, y_{s^{-}}, \theta_{s^{-}}, \boldsymbol{x}_{s^{-}}+\boldsymbol{x}_{s^{-}} \boldsymbol{G}\left(s, y_{s^{-}}, \theta_{s^{-}}, \boldsymbol{x}_{s^{-}}, z\right)\right)-V\left(s, y_{s^{-}}, \theta_{s^{-}}, \boldsymbol{x}_{s^{-}}\right)\right] \bar{\psi}\left(\theta_{s^{-}}, d z, d s\right) \\
& +\int_{|z|>1}\left[V\left(s, y_{s^{-}}, \theta_{s^{-}}, \boldsymbol{x}_{s^{-}}+\boldsymbol{H}\left(s, y_{s^{-}}, \theta_{s^{-}}, \boldsymbol{x}_{s^{-}}, z\right)\right)-V\left(s, y_{s^{-}}, \theta_{s^{-}}, \boldsymbol{x}_{s^{-}}\right)\right] \psi\left(\theta_{s^{-}}, d z, d s\right) \\
& \int_{|z| \leq 1}\left[V\left(s, y_{s^{-}}, \theta_{s^{-}}, \boldsymbol{x}_{s^{-}}+\boldsymbol{x}_{s^{-}} \boldsymbol{G}\left(s, y_{s^{-}}, \theta_{s^{-}}, \boldsymbol{x}_{s^{-}}, z\right)\right)-V\left(s, y_{s^{-}}, \theta_{s^{-}}, \boldsymbol{x}_{s^{-}}\right)\right. \\
& \left.-\frac{\partial V}{\partial x} \boldsymbol{G}\left(s, y_{s^{-}}, \theta_{s^{-}}, \boldsymbol{x}_{s^{-}}, z\right)\right] v\left(\theta_{s^{-}}, d z\right) d s \\
& +\sum_{j=1, j \neq \theta_{s^{-}}}^{m} \int_{z \in \mathbb{R}}\left[V\left(s, y_{s}, j, \boldsymbol{x}_{s^{-}} e^{z}\right)-V\left(s, y_{s^{-}}, \theta_{s^{-}}, \boldsymbol{x}_{s^{-}}\right)\right] N\left(d z, d s,\left\{\left(\theta_{s^{-}}, j\right)\right\}\right) \\
& =\mathcal{L} V\left(s, \theta_{s}, y_{s}, \boldsymbol{x}_{s}\right) d s+\frac{\partial V}{\partial \boldsymbol{x}} \boldsymbol{\sigma}\left(s, \theta_{k}, y_{s^{-}}, \boldsymbol{x}_{s^{-}}\right) d \boldsymbol{B}_{s} \\
& +\int_{|z| \leq 1}\left[V\left(s, y_{s^{-}}, \theta_{s^{-}}, \boldsymbol{x}_{s^{-}}+\boldsymbol{G}\left(s, y_{s^{-}}, \theta_{s^{-}}, \boldsymbol{x}_{s^{-}}, z\right)\right)-V\left(s, y_{s^{-}}, \theta_{s^{-}}, \boldsymbol{x}_{s^{-}}\right)\right] \bar{\psi}\left(\theta_{s^{-}}, d z, d s\right) \\
& \int_{|z|>1}\left[V\left(s, y_{s^{-}}, \theta_{s^{-}}, \boldsymbol{x}_{s^{-}}+\boldsymbol{H}\left(s, y_{s^{-}}, \theta_{s^{-}}, \boldsymbol{x}_{s^{-}}, z\right)\right)-V\left(s, y_{s^{-}}, \theta_{s^{-}}, \boldsymbol{x}_{s^{-}}\right)\right] \bar{\psi}\left(\theta_{s^{-}}, d z, d s\right) \\
& +\sum_{j=1, j \neq \theta_{s^{-}}}^{m} \int_{z \in \mathbb{R}}\left[V\left(s, y_{s}, j, \boldsymbol{x}_{s^{-}} e^{z}\right)-V\left(s, y_{s^{-}}, \theta_{s^{-}}, \boldsymbol{x}_{s^{-}}\right)\right] \bar{N}\left(d z, d s,\left\{\left(\theta_{s^{-}}, j\right)\right\}\right),
\end{aligned}
$$

where, 


$$
\begin{aligned}
& \mathcal{L} V\left(s, \theta_{s^{-}}, y_{s^{-}}, \boldsymbol{x}_{s^{-}}\right)=\frac{\partial V}{\partial s}+\frac{\partial V}{\partial y}+\frac{\partial V}{\partial \boldsymbol{x}} \boldsymbol{\mu}\left(t, \theta_{k}, y_{s^{-}}, \boldsymbol{x}_{s^{-}}\right) \\
& +\frac{1}{2} \operatorname{Tr}\left[\boldsymbol{\sigma}^{T}\left(s, y_{s^{-}}, \theta_{s^{-}}, \boldsymbol{x}_{s^{-}}\right) \frac{\partial^{2} V}{\partial \boldsymbol{x}^{2}} \boldsymbol{\sigma}\left(s, y_{s^{-}}, \theta_{s^{-}}, \boldsymbol{x}_{s^{-}}\right)\right] \\
& +\int_{|z| \leq 1}\left[V\left(s, y_{s^{-}}, \theta_{s^{-}}, \boldsymbol{x}_{s^{-}}+\boldsymbol{G}\left(s, y_{s^{-}}, \theta_{s^{-}}, \boldsymbol{x}_{s^{-}}, z\right)\right)-V\left(s, y_{s^{-}}, \theta_{s^{-}}, \boldsymbol{x}_{s^{-}}\right)\right. \\
& \left.-\frac{\partial V}{\partial \boldsymbol{x}} \boldsymbol{G}\left(s, y_{s^{-}}, \theta_{s^{-}}, \boldsymbol{x}_{s^{-}}, z\right)\right] v\left(\theta_{s^{-}}, d z\right) \\
& +\int_{|z|>1}\left[V\left(s, y_{s^{-}}, \theta_{s^{-}}, \boldsymbol{x}_{s^{-}}+\boldsymbol{H}\left(s, y_{s^{-}}, \theta_{s^{-}}, \boldsymbol{x}_{s^{-}}, z\right)\right)-V\left(s, y_{s^{-}}, \theta_{s^{-}}, \boldsymbol{x}_{s^{-}}\right)\right] v\left(\theta_{s^{-}}, d z\right) \\
& +\int_{z \in \mathbb{R}} \sum_{j \in E-\left\{\theta_{s^{-}}\right.}\left[V\left(s, y_{s}, j, \boldsymbol{x}_{s^{-}} e^{z}\right)-V\left(s, y_{s^{-}}, \theta_{s^{-}}, \boldsymbol{x}_{s^{-}}\right)\right] \gamma\left(d z, d s,\left\{\left(\theta_{s^{-}}, j\right)\right\}\right)
\end{aligned}
$$

We denote $P_{M^{n}}^{n}=\left\{t_{k}\right\}_{k=0}^{M_{n}}, 0=t_{0}<t_{1}<\ldots<t_{M_{n}}=T$ a partition of the time interval [0,T]. Using the notations

$$
\begin{aligned}
& V_{k}=V\left(t_{k}, \theta_{t_{k}}, y_{t_{k}}, \boldsymbol{x}_{t_{k}}\right), G_{k}=G\left(t_{k}, y_{t_{k}}, \theta_{t_{k}}, \boldsymbol{x}_{t_{k}}, z\right), \\
& H_{k}=H\left(t_{k}, y_{t_{k}}, \theta_{t_{k}}, \boldsymbol{x}_{t_{k}}, z\right), \\
& V_{k-1}^{G}(z)=V\left(t_{k-1}, y_{t_{k-1}}, \theta_{t_{k-1}}, x_{t_{k-1}}+\boldsymbol{G}\left(t_{k-1}, y_{t_{k-1}}, \theta_{t_{k-1}}, \boldsymbol{x}_{t_{k-1}}, z\right)\right), \\
& V_{k-1}^{H}(z)=V\left(t_{k-1}, y_{t_{k-1}}, \theta_{t_{k-1}}, x_{t_{k-1}}+\boldsymbol{H}\left(t_{k-1}, y_{t_{k-1}}, y_{t_{k-1}}, \boldsymbol{x}_{t_{k-1}}, z\right)\right) .
\end{aligned}
$$

The first and second moments are presented below:

$$
\begin{aligned}
& E\left[\Delta V_{k} \mid \mathbb{G}_{t_{k-1}}\right]=\mathcal{L} V_{k-1} \Delta t_{k} \\
& E\left[\left[\Delta V_{k}-E\left[\Delta V_{k} \mid \mathbb{G}_{t_{k-1}}\right]\right]\left[\Delta V_{k}-E\left[\Delta V_{k} \mid \mathbb{G}_{t_{k-1}}\right]\right]^{T} \mid \mathbb{G}_{t_{k-1}}\right] \\
& =\frac{\partial V}{\partial \boldsymbol{x}} \boldsymbol{\sigma}\left(s, y_{s^{-}}, \theta_{s^{-}}, \boldsymbol{x}_{s^{-}}\right) \boldsymbol{\sigma}^{T}\left(s, y_{s^{-}}, \theta_{s^{-}}, \boldsymbol{x}_{s^{-}}\right) \frac{\partial V^{T}}{\partial \boldsymbol{x}} \Delta t_{k-1} \\
& +\int_{|z| \leq 1}\left[V_{k-1}^{G}(z)-V_{k-1}\right]\left[V_{k-1}^{G}(z)-V_{k-1}\right]^{T} v\left(\theta_{t_{k-1}}, d z\right) \Delta t_{k} \\
& +\int_{|z|>1}\left[V_{k-1}^{H}(z)-V_{k-1}\right]\left[V_{k-1}^{H}(z)-V_{k-1}\right]^{T} v\left(\theta_{t_{k-1}}, d z\right) \Delta t_{k} \\
& +\sum_{j \in E-\left\{\theta_{\left.t_{k-1}\right\}}\right.} \int_{z \in \mathbb{R}}\left[V\left(t_{k-1}, y_{t_{k-1}}, j, \boldsymbol{x}_{t_{k-1}} e^{z}\right)-V_{k-1}\right]\left[V\left(t_{k-1}, y_{t_{k-1}}, j, \boldsymbol{x}_{t_{k-1}} e^{z}\right)-V_{k-1}\right]^{T} \\
& \times \gamma\left(d z, \Delta t_{k},\left\{\left(\theta_{t_{k-1}}, j\right)\right\}\right)
\end{aligned}
$$

\section{Illustrations}

In the following, we examine two particular cases of the model in (2).

\subsection{Modeling of a Basket of Interdependent Assets}

We extend the system of interconnected commodity price process network in Otunuga and Ladde (2014) by considering Levy jumps representing shocks specific to each member of the network. We assume that each asset in the network is affected by independent unpredictable shocks/informations. If the network considered is a network of financial assets, unpredictable shocks may originate from sudden information affecting investors views of the particular financial sector of interest or other unpredictable change affecting parameters playing a preeminent role on the asset price. Unlike and Ladde (2014); Otunuga et al. (2016), we assume two layers of interactions of asset prices in the network. We assume asset price interactions in the diffusion coefficient and in the price jumps. Similarly to Otunuga and Ladde (2014); Otunuga et al. (2016), the $\mathrm{i}$-th asset price impacts the $\mathrm{j}$-th asset price through cross diffusion coefficients of order $(\mathrm{i}, \mathrm{j})$. A second layer of interaction introduced in this paper is achieved by assuming that a jump of the $\mathrm{i}$-th asset price impacts the $\mathrm{j}$-th asset price through an appropriately modeled cross dependence parameter of order $(\mathrm{i}, \mathrm{j})$. Let us assume that the network entails $n \geq 1$ assets. We model these shocks with a family of Lévy-Itó processes with Poisson measures $\psi^{m}=\left(\psi_{1}^{m}, \ldots \psi_{n}^{m}\right)^{T}$ having intensity processes $\boldsymbol{v}^{m}=\left(v_{1}^{m}, \ldots \nu_{n}^{m}\right)^{T}$ and $\boldsymbol{\psi}^{p}=\left(\psi_{1}^{p}, \ldots \psi_{n}^{p}\right)^{T}$ having intensity processes $\boldsymbol{v}^{p}=\left(v_{1}^{p}, \ldots v_{n}^{p}\right)^{T}$. We also 
denote $\bar{\psi}^{m}=\left(\bar{\psi}_{1}^{m}, \ldots \bar{\psi}_{n}^{m}\right)^{T}=\psi^{m}-\boldsymbol{v}^{m}$ and $\boldsymbol{\psi}^{p}=\left(\psi_{1}^{p}, \ldots \psi_{n}^{p}\right)^{T}$ with intensity processes $\boldsymbol{v}^{p}=\left(v_{1}^{p}, \ldots v_{n}^{p}\right)^{T}$. We assume that the vector mean process $\boldsymbol{m}^{m}=\left(m_{1}^{m}, \ldots m_{n}^{m}\right)$ and vector price process $\boldsymbol{p}^{m}=\left(p_{1}^{m}, \ldots p_{n}^{m}\right)$ potentially react differently to market shocks through Poisson integrands $\left(G_{i}^{m}\right)_{i=1}^{n},\left(H_{i}^{m}\right)_{i=1}^{n},\left(G_{i}^{p}\right)_{i=1}^{n}$ and $\left(H_{i}^{p}\right)_{i=1}^{n}$. We introduce the following matrices: $\boldsymbol{G}^{m}=\left(G_{j}^{m}\right)_{j \leq n}, \boldsymbol{G}^{p}=\left(G_{j}^{p}\right)_{j \leq n}, \boldsymbol{H}^{m}=\left(H_{j}^{m}\right)_{j \leq n}, \boldsymbol{G}^{p}=\left(H_{j}^{p}\right)_{j \leq n}, \boldsymbol{W}=\left(W_{i, j}\right)_{i, j \leq n}$ and and $\boldsymbol{Z}=\left(Z_{i, j}\right)_{i, j \leq n}$ are real numbers $\forall i, j \in I(1, n) . W_{i, j}$ and $Z_{i, j}$ are independent Brownian motion processes. A coupled system of Levy type stochastic differential equations under regime changes and subjected to structural perturbations can be expressed as follows:

$$
\left\{\begin{array}{l}
d m_{j}=\left(u_{j}-m_{j}\right)\left[\sum_{l=1}^{n} \kappa_{l j}\left(t^{-}\right) m_{l}\left(t^{-}\right) d t+\delta_{j j} d W_{j, j}(t)+\sum_{l=1, l \neq j}^{n} \delta_{l j} m_{l}\left(t^{-}\right) d W_{l, j}(t)\right] \\
+\int_{|l|<1} G_{j}^{m}\left(t, \theta_{t^{-}}, y_{t^{-}}, m_{t^{-}}, z\right) \bar{\psi}_{j}^{m}\left(\theta_{t^{-}}, d t, d z\right)+\int_{|z|>1} H_{j}^{m}\left(t, \theta_{t^{-}}, y_{t^{-}}, m_{t}, z\right) \psi_{j}^{m}\left(\theta_{t^{-}}, d t, d z\right), t \in\left[T_{k}, T_{k+1}\right), m_{j}\left(t_{0}\right)=m_{j, 0}, \\
m_{j}\left(T_{k}\right)=m_{j}^{k} \text { and } m_{j}^{k}=\pi_{j}^{k} m\left(T_{k}^{-}, T_{k-1}, m\right), \forall k \in I(1, \infty), \\
d p_{j}=p_{j}\left[\left[\gamma_{j j}\left(m_{j}-p_{j}\right)+\beta_{j}+\sum_{l=1, l \neq j}^{n} \gamma_{l j}\left(t^{-}\right) p_{l}\left(t^{-}\right)\right] d t+\sigma_{j j} d Z_{j, j}(t)+\sum_{l=1, l \neq j}^{n} \sigma_{l j} p_{l}\left(t^{-}\right) d Z_{l, j}(t)\right] \\
+\int_{|z|<1} G_{j}^{p}\left(t, \theta_{t^{-}}, y_{t^{-}}, p_{t^{-}}, z\right) \bar{\psi}_{j}^{p}\left(\theta_{t^{-}}, d t, d z\right)+\int_{|l|>1} H_{j}^{p}\left(t, \theta_{t^{-}}, y_{t^{-}}, p_{t}, z\right) \psi_{j}^{p}\left(\theta_{t^{-}}, d t, d z\right), t \in\left[T_{k}, T_{k+1}\right), p_{j}\left(t_{0}\right)=p_{j, 0}, \\
p_{j}\left(T_{k}\right)=p_{j}^{k} \text { and } p_{j}^{k}=\omega_{j}^{k} p\left(T_{k}^{-}, T_{k-1}, p, m\right), \forall k \in I(1, \infty) .
\end{array}\right.
$$

We define the following matrices:

$$
\begin{aligned}
& \boldsymbol{\kappa}=\left(\begin{array}{ccc}
\kappa_{11} & \ldots & \kappa_{1 n} \\
\vdots & \ldots & \vdots \\
\kappa_{n 1} & \ldots & \kappa_{n n}
\end{array}\right), \boldsymbol{\gamma}=\left(\begin{array}{ccc}
-\gamma_{11} & \ldots & \gamma_{1 n} \\
\vdots & \ldots & \vdots \\
\gamma_{n 1} & \ldots & -\gamma_{n n}
\end{array}\right), \\
& \boldsymbol{\Sigma}=\left(\begin{array}{ccc}
\sigma_{11} & \ldots & \sigma_{1 n} p_{1} \\
\vdots & \ldots & \vdots \\
\sigma_{n 1} p_{n} & \ldots & \sigma_{n n}
\end{array}\right), \boldsymbol{\Upsilon}=\left(\begin{array}{ccc}
\delta_{11} & \ldots & \delta_{1 n} m_{1} \\
\vdots & \ldots & \vdots \\
\delta_{n 1} m_{n} & \ldots & \delta_{n n}
\end{array}\right), \\
& \boldsymbol{W}=\left(\begin{array}{ccc}
W_{11} & \ldots & W_{1 n} \\
\vdots & \ldots & \vdots \\
W_{n 1} & \ldots & W_{n n}
\end{array}\right), \boldsymbol{Z}=\left(\begin{array}{ccc}
Z_{11} & \ldots & Z_{1 n} \\
\vdots & \ldots & \vdots \\
Z_{n 1} & \ldots & Z_{n n}
\end{array}\right)
\end{aligned}
$$

This coupled system in matrix form becomes:

$$
\begin{aligned}
& d \boldsymbol{m}_{t}=\boldsymbol{a}\left(t, y_{t}, \theta_{j}, \boldsymbol{m}\right) d t+\Upsilon\left(t, y_{t}, \theta_{j}, \boldsymbol{m}\right) d \boldsymbol{W}(t)+\int_{|z|<1} \boldsymbol{G}^{m}\left(t, y_{t}, \theta_{j}, \boldsymbol{m}, z\right) \bar{\psi}_{m}\left(\theta_{j}, d t, d z\right) \\
& +\int_{|z|>1} \boldsymbol{H}^{m}\left(t, y_{t}, \theta_{t}, \boldsymbol{m}, z\right) \psi\left(\theta_{j}, d t, d z\right), \text { where } \boldsymbol{m}\left(t_{0}\right)=\boldsymbol{m}_{0}, \text { if } t \in\left[T_{j}, T_{j+1}\right) \\
& d \boldsymbol{p}_{t}=\boldsymbol{b}\left(t, y, \theta_{j}, \boldsymbol{p}, \boldsymbol{m}\right) d t+\boldsymbol{\Sigma}\left(t, y, \theta_{j}, \boldsymbol{p}\right) d \boldsymbol{Z}(t)+\int_{|z|<1} \boldsymbol{G}^{p}\left(t, y, \theta_{j}, \boldsymbol{p}, z\right) \bar{\psi}_{p}\left(\theta_{j}, d t, d z\right) \\
& +\int_{|z|>1} \boldsymbol{H}^{p}\left(t, y_{t}, \theta_{j}, \boldsymbol{p}, z\right) \psi\left(\theta_{j}, d t, d z\right), \text { where } \boldsymbol{p}\left(t_{0}\right)=\boldsymbol{p}_{0}, \text { if } t \in\left[T_{j}, T_{j+1}\right) \\
& \boldsymbol{m}^{i}=\Pi^{i} \boldsymbol{m}^{i-1}\left(T_{i}^{-}, T_{i-1}, \boldsymbol{m}^{i-1}\right), \\
& \boldsymbol{p}^{i}=\Omega^{i} \boldsymbol{p}^{i-1}\left(T_{i}^{-}, T_{i-1}, \boldsymbol{p}^{i-1}\right), \\
& \text { with } \\
& \Pi^{i}=\operatorname{diag}\left(\pi_{1}, \ldots, \pi_{n}\right), \text { where } \ln \left(\pi_{k}\right) \sim g\left(\mid \theta_{i}, \theta_{i-1}\right), \forall k \in I(1, n), i \in I(1, \infty), \\
& \Omega^{i}=\operatorname{diag}\left(\omega_{1}, \ldots \omega_{n}\right), \text { where } \ln \left(\omega_{k}\right) \sim h\left(\mid \theta_{i}, \theta_{i-1}\right), \forall k \in I(1, n), i \in I(1, \infty),
\end{aligned}
$$

where $\boldsymbol{a}\left(t, y, \theta_{j}, \boldsymbol{m}\right)$ and $\boldsymbol{b}\left(t, y, \theta_{j}, \boldsymbol{p}, \boldsymbol{m}\right)$ are $n$ dimensional vectors with $\boldsymbol{a}\left(t, y, \theta_{j}, \boldsymbol{p}, \boldsymbol{m}\right)=\boldsymbol{\kappa}^{T}\left(t^{-}\right) \boldsymbol{p}\left(t^{-}\right)$and $\boldsymbol{b}\left(t, y, \theta_{j}, \boldsymbol{p}, \boldsymbol{m}\right)=$ $-\gamma \operatorname{diag}\left(m_{1}, \ldots, m_{n}\right)+\boldsymbol{\beta}+\boldsymbol{\gamma} \boldsymbol{p}$; We consider the particular case of (13) where,

$$
\begin{aligned}
& H_{l}^{p}\left(t, y_{t}, \theta_{t}, \boldsymbol{p}_{t}, z\right)=G_{l}^{p}\left(t, y_{t}, \theta_{t}, \boldsymbol{p}_{t}, z\right)=z \\
& H_{l}^{m}\left(t, y_{t}, \theta_{t}, \boldsymbol{m}_{t}, z\right)=G_{l}^{m}\left(t, y_{t}, \theta_{t}, \boldsymbol{p}_{t}, z\right)=z .
\end{aligned}
$$

We also consider the following families of Lyapunov functions: $V^{j, q}\left(t, y_{t}, \theta_{t}, \boldsymbol{m}\right)=m_{j}^{q}$ and $V^{j, q}\left(t, y_{t}, \theta_{t}, \boldsymbol{p}\right)=p_{j}^{q} \forall j \in$ $I(1, n), q \in I(1, \infty)$. Using the multivariate real valued version of Itó formula in (9) and (11) applied to $U$, we obtain, 
respectively:

$$
\begin{aligned}
& d\left(m_{j}^{q}(t)\right)=q m_{j}^{q-1}\left(t^{-}\right)\left(u_{j}-m_{j}\left(t^{-}\right)\right)\left[\sum_{l=1}^{n} \kappa_{j, l} m_{l}\left(t^{-}\right)\right] d t \\
& +\frac{1}{2} q(q-1) m_{j}^{q-2}\left(t^{-}\right)\left(u_{j}-m_{j}\left(t^{-}\right)\right)^{2}\left[\delta_{j, j}^{2}+\sum_{l=1, l \neq j}^{n} \delta_{j, l}^{2} m_{l}^{2}\left(t^{-}\right)\right] d t \\
& +\int_{|z| \leq 1}\left[\left[m_{j}\left(t^{-}\right)+m_{j}\left(t^{-}\right) G_{l}^{m}\left(t^{-}\right)\right]^{q}-m_{j}^{q}\left(t^{-}\right)\right] \bar{\psi}_{j}^{m}\left(\theta_{t^{-}}, d z\right) \\
& +\int_{|z|>1}\left[\left[m_{j}^{q}\left(t^{-}\right)+m_{j}\left(t^{-}\right) H_{j}^{m}\left(t^{-}\right)\right]^{q}-m_{j}^{q}\left(t^{\prime}\right] \bar{\psi}_{j}^{m}\left(\theta_{t^{-}}, d z\right)\right. \\
& \int_{|z| \leq 1}\left[\left[m_{j}\left(t^{-}\right)+m_{j}\left(t^{-}\right) G_{l}^{m}\left(t^{-}\right)\right]^{q}-m_{j}^{q}\left(t^{-}\right)-q m_{j}^{q}\left(t^{-}\right) G_{m}^{j}\right] v_{j}^{m}\left(\theta_{t^{-}}, d z\right) \\
& +\int_{|z|>1}\left[\left[m_{j}^{q}\left(t^{-}\right)+m_{j}\left(t^{-}\right) H_{j}^{l}\left(t^{-}\right)\right]^{q}-m_{j}^{q}\left(t^{\prime}\right) v_{j}^{m}\left(\theta_{t^{-}}, d z\right)\right. \\
& +q m^{q-1}\left(t^{-}\right)\left(u_{j}-m_{j}\left(t^{-}\right)\right)\left[\delta_{j, j} d W_{j, j}(t)+\sum_{l=1, l \neq j}^{n} \delta_{j, l} m_{l}\left(t^{-}\right) d W_{j, l}(t)\right], \forall t \in\left[T_{s}, T_{s+1}\right], s \in I(1, \infty), \\
& d\left(p_{j}^{q}(t)\right)=q p_{j}^{q}\left(t^{-}\right)\left[\gamma_{j, j}\left(m_{j}\left(t^{-}\right)-p_{j}\left(t^{-}\right)\right)+\beta_{j}+\sum_{l=1}^{n} \gamma_{j, l} p_{l}(t)\right] d t \\
& +\frac{1}{2} q(q-1) p_{j}^{q-2}\left(t^{-}\right) p_{j}^{2}\left(t^{-}\right)\left[\sigma_{j, j}^{2}+\sum_{l=1, l \neq j}^{n} \sigma_{j, l}^{2} p_{l}^{2}\left(t^{-}\right)\right] \\
& +\int_{|z| \leq 1}\left[\left[p_{j}\left(t^{-}\right)+p_{j}\left(t^{-}\right) G_{j}^{p}\left(t^{-}\right)\right]^{q}-p_{j}^{q}\left(t^{-}\right)\right] \bar{\psi}_{j}^{p}\left(\theta_{t^{-}}, d z\right) \\
& +\int_{|z|>1}\left[\left[p_{j}\left(t^{-}\right)+p_{j}\left(t^{-}\right) H_{j}^{p}\left(t^{-}\right)\right]^{q}-p_{j}^{q}\left(t^{-}\right)\right] \bar{\psi}_{p}^{j}\left(\theta_{t^{-}}, d z\right) \\
& \int_{|z| \leq 1}\left[\left[p_{j}\left(t^{-}\right)+p_{j}\left(t^{-}\right) G_{j}^{p}\right]^{q}-p_{j}^{q}\left(t^{-}\right)-q p^{q}\left(t^{-}\right) G_{p}^{j}\right] v_{j}^{p}\left(\theta_{t^{-}}, d z\right) \\
& +\int_{|z|>1}\left[\left[p_{j}\left(t^{-}\right)+p_{j}\left(t^{-}\right) H_{j}^{p}\right]^{q}-p_{j}^{q}\left(t^{-}\right)\right] v_{p}^{j}\left(\theta_{t^{-}}, d z\right) \\
& +q p^{q}\left(t^{-}\right)\left[\sigma_{j, j} d Z_{j, j}+\sum_{l=1, l \neq j}^{n} \sigma_{j, l} p_{l}\left(t^{-}\right) d Z_{j, l}\left(t^{-}\right)\right], \forall t \in\left[T_{s}, T_{s+1}\right], s \in I(1, \infty) .
\end{aligned}
$$

Applying Euler Maruyama discretization scheme at $t_{k}$ leads to:

$$
\begin{aligned}
& \left.E\left[\Delta m_{j}^{q}\left(t_{k}\right) \mid \mathbb{G}_{t_{k-1}}\right]=q m_{j}^{q-1}\left(t_{k-1}\right)\left(u_{j}-m_{j}\left(t_{k-1}\right)\right)\left[\sum_{l=1}^{n} \kappa_{j, l} m_{l}\left(t^{-}\right)\right)\right] \Delta t_{k} \\
& +\frac{1}{2} q(q-1) m_{j}^{q-2}\left(t_{k-1}\right)\left(u_{j}-m_{j}\left(t_{k-1}\right)\right)^{2}\left[\delta_{j, j}^{2}\left(t_{k-1}\right)+\sum_{l=1, l \neq j}^{2} \delta_{j, l}^{2}\left(t_{k-1}\right) m_{l}^{2}\left(t_{k-1}\right)\right] \\
& +\int_{|k| \leq 1}\left[\left[m_{j}\left(t_{k-1}\right)+m_{j}\left(t^{-}\right) G_{j}^{m}\left(t_{k-1}\right)\right]^{q}-m_{j}^{q}\left(t_{k-1}\right)-q m_{j}^{q}\left(t_{k-1}\right) G_{j}^{m}\left(t_{k-1}\right)\right] v_{j}^{m}\left(\theta_{t_{k-1}}, d z\right) \\
& +\int_{|z|>1}\left[\left[m_{j}\left(t_{k-1}\right)+m_{j}\left(t^{-}\right) H_{j}^{m}\left(t_{k-1}\right)\right]^{q}-m_{j}^{q}\left(t_{k-1}\right)\right] v_{j}^{m}\left(\theta_{s^{-}}, d z\right),
\end{aligned}
$$




$$
\begin{aligned}
& E\left[\left[\Delta m_{j}^{q}\left(t_{k}\right)-E\left[\Delta m_{j}^{q}\left(t_{k}\right) \mid \mathbb{G}_{t_{k-1}}\right]\right]^{2} \mid \mathbb{G}_{t_{k-1}}\right] \\
& =\left(u_{j}-m_{j}\left(t_{k-1}\right)\right)^{2}\left[q^{2} m_{j}^{2(q-1)}\left(t_{k-1}\right)\left[\delta_{j, j}^{2}+\sum_{l=1, l \neq j}^{n} \delta_{j, l}^{2} m_{l}^{2}\left(t_{k-1}\right)\right] \Delta t_{k}\right. \\
& +\int_{|z| \leq 1}\left[\left[m_{j}\left(t_{k-1}\right)+m_{j}\left(t^{-}\right) G_{m}^{j}\left(t_{k-1}\right)\right]^{q}-m_{j}^{q}\left(t_{k-1}\right)\right]^{2} v_{j}^{m}\left(\theta_{t_{k-1}}, d z\right) \Delta t_{k} \\
& \left.\left.+\int_{|z|>1}\left[\left[m_{j}\left(t_{k-1}\right)+m_{j}\left(t^{-}\right) H_{m}^{j}\left(t_{k-1}\right)\right]^{q}-m_{j}^{q}\left(t_{k-1}\right)\right]^{2} v_{j}^{m}\left(\theta_{t_{k-1}}, d z\right) \Delta t_{k}\right]\right],
\end{aligned}
$$

and

$$
\begin{aligned}
& E\left[\Delta p_{j}^{q}\left(t_{k}\right) \mid \mathbb{G}_{t_{k-1}}\right]=q p_{j}^{q}\left(t_{k-1}\right)\left[\gamma_{j, j}\left(m_{j}\left(t^{-}\right)-p_{j}\left(t^{-}\right)\right)+\beta_{j}+\sum_{l=1}^{n} \gamma_{j, l} p_{l}(t)\right] \Delta t_{k} \\
& +\frac{1}{2} q(q-1) p_{j}^{q}\left(t_{k}\right)\left[\sigma_{j, l}^{2}+\sum_{l=1, l \neq j}^{n} \sigma_{j, l}^{2} p_{l}^{2}\left(t_{k-1}\right)\right] \\
& +\int_{|z| \leq 1}\left[\left[p_{j}\left(t_{k-1}\right)+p_{j}\left(t_{k-1}\right) G_{j}^{p}\left(t_{k-1}\right)\right]^{q}-p_{j}^{q}\left(t_{k-1}\right)-q p_{j}^{q}\left(t_{k-1}\right) G_{j}^{p}\left(t_{k-1}\right)\right] v_{j}^{p}\left(\theta_{s^{-}}, d z\right) \\
& +\int_{|z|>1}\left[\left[p_{j}\left(t_{k-1}\right)+p_{j}\left(t_{k-1}\right) H_{j}^{p}\left(t_{k}\right)\right]^{q}-p_{j}^{q}\left(t_{k-1}\right)\right] v_{j}^{p}\left(\theta_{s^{-}}, d z\right), \\
& E\left[\left[\Delta p_{j}^{q}\left(t_{k}\right)-E\left[\Delta p_{j}^{q}\left(t_{k}\right) \mid \mathbb{G}_{t_{k-1}}\right]\right]^{2} \mid \mathbb{G}_{t_{k-1}}\right]=q^{2} p_{j}^{2} q\left(t_{k-1}\right)\left[p_{j}^{2}\left(t_{k-1}\right)+\sum_{l=1, l \neq j}^{n} \sigma_{j, l}^{2}\left(t_{k-1}\right)\right] \Delta t_{k} \\
& +\int_{|z| \leq 1}\left[\left[p_{j}\left(t_{k-1}\right)+p_{j}\left(t_{k-1}\right) G_{l}^{p}\left(t_{k-1}\right)\right]^{q}-p_{j}^{q}\left(t_{k-1}\right)\right]^{2} v_{j}^{p}\left(\theta_{t_{k-1}}, d z\right) \Delta t_{k} \\
& +\int_{|z|>1}\left[\left[p_{j}\left(t_{k-1}\right)+p_{j}\left(t_{k-1}\right) H_{j}^{p}\left(t_{k-1}\right)\right]^{q}-p_{j}^{q}\left(t_{k-1}\right)\right]^{2} v_{j}^{p}\left(\theta_{t_{k-1}}, d z\right) \Delta t_{k} .
\end{aligned}
$$

We note that Levy jumps sizes of a fixed price process(resp: fixed mean process) are independent and identically distributed for a given market regime. The Euler Maruyama discretization in (21) and (23) involve first and second moments of Levy jump sizes which we will estimate using Monte Carlo integration. We note from model definition in 13 that the contribution of small and big Levy jumps in $d p_{j}(t)$ (resp: $m_{j}(t)$ ), the price (resp: the mean) change in asset $j$ at time $t$ are respectively $p_{j}\left(t^{-}\right) \int_{|z|<1} G_{l}^{p}\left(t, \boldsymbol{p}_{t^{-}}, z\right)$ and $p_{j}\left(t^{-}\right) \int_{|z|>1} H_{l}^{p}\left(t, \boldsymbol{p}_{t}, z\right)\left(\right.$ resp: $m_{j}\left(t^{-}\right) \int_{|z|<1} G_{l}^{m}\left(t, \boldsymbol{m}_{t^{-}}, z\right)$ and $\left.m_{j}\left(t^{-}\right) \int_{|z|>1} H_{l}^{m}\left(t, \boldsymbol{m}_{t}, z\right)\right)$. Hence, the Levy Jumps are estimated from the price and mean process return time series in the following manner (see Robert and Casella (2013)):

$$
\int_{s}^{t} \int_{z \in \mathbb{R}} f(\eta, z) v_{l}^{m}(i, d z) d \eta \approx \frac{1}{n^{l}(i, m,[s, t])} \sum_{\zeta_{i}^{m_{l} \in[s, t]}}^{n\left(m_{l}[s, t]\right)} f\left(\zeta_{i}^{m_{l}}, z_{\zeta_{i}} \xi_{l}\right), \forall t, s \in[0, T] \text { with } \theta_{\eta}=i, \forall \eta \in[s, t] .
$$

$\left(\zeta_{i}^{m_{l}}\right)_{i \in \mathcal{N}^{\star}}$ is the sequence of Levy time jumps of the mean price process $m_{l} . n\left(m_{l},[s, t]\right)$ denotes the total number of jumps of the $l-t h$ mean asset price process while in regime $i$ in the time interval $[s, t]$ and $\left(z_{\zeta_{i}^{m}}\right)_{i \in \mathcal{N}^{*}}$ represents the corresponding sequence of Levy jump sizes. We assume that the function $f$ is integrable with respect to the product measure $v_{l}^{m}(i) d$,$t in$ the domain $\mathbb{R} \times[0, T]$. Throughout the discretization process. the four functions

$$
\begin{aligned}
& f(\eta, z)=1_{(|k| \leq 1)}\left[\left[p_{j}\left(t_{k-1}\right)+p_{j}\left(t_{k-1}\right) G_{j}^{p}\left(t_{k-1}, z\right)\right]^{q}-p_{t_{k-1}}^{q}\right]^{2}, \\
& g(\eta, z)=1_{(|k| \leq 1)}\left[\left[p_{j}\left(t_{k-1}\right)+p_{j}\left(t_{k-1}\right) G_{j}^{p}\left(t_{k-1}, z\right)\right]^{q}-p_{t_{k-1}}^{q}-q p_{j}^{q}\left(t_{k-1}\right) G_{j}^{p}\left(t_{k-1}, z\right)\right], \\
& h(\eta, z)=1_{(|k|>1)}\left[\left[p_{j}\left(t_{k-1}\right)+p_{j}\left(t_{k-1}\right) H_{j}^{p}\left(t_{k-1}, z\right)\right]^{q}-p_{t_{k-1}}^{q}\right]^{2}, \\
& s(\eta, z)=1_{(|k|>1)}\left[\left[p_{j}\left(t_{k-1}\right)+p_{j}\left(t_{k-1}\right) H_{j}^{p}\left(t_{k-1}, z\right)\right]^{q}-p_{t_{k-1}}^{q}\right],
\end{aligned}
$$

are integrated with respect to $v_{j}^{p}$. Similarly, the four counterparts of the functions in (26) with the price function replaced by the mean function are integrated with respect to $v_{j}^{m}$. Those integrals are approximated as described by the Monte Carlo integral (25). For convenience of notation, from here on, for each function $\mathrm{f}, \mathrm{g}, \mathrm{h}$ and $\mathrm{s}$ in (26) we denote the Monte Carlo integral estimates in any time period $\left[t_{k}, t_{k+1}\right]$ by respectively by $M^{2}(s p), M^{1}(s p), M^{2}(b p)$ and $M^{1}(b p)$. If the mean process 
is used in (26) similar notations are used with $m$ substituting $p$.

$$
\begin{aligned}
& \left.E\left[\Delta m_{j}^{q}\left(t_{k}\right) \mid \mathbb{G}_{t_{k-1}}\right]=q m_{j}^{q-1}\left(t_{k-1}\right)\left(u_{j}-m_{j}\left(t_{k-1}\right)\right)\left[\sum_{l=1}^{n} \kappa_{j, l} m_{l}\left(t^{-}\right)\right)\right] \Delta t_{k} \\
& +\frac{1}{2} q(q-1) m_{j}^{q-2}\left(t_{k-1}\right)\left(u_{j}-m_{j}\left(t_{k-1}\right)\right)^{2}\left[\sum_{l=1}^{2} \delta_{j, l}^{2}\left(t_{k-1}\right)\right]+q m_{j}^{q}\left(t^{-}\right) M_{j}^{1}(b m), \\
& E\left[\left[\Delta m_{j}^{q}\left(t_{k}\right)-E\left[\Delta m_{j}^{q}\left(t_{k}\right) \mid \mathbb{G}_{t_{k-1}}\right]\right]^{2} \mid \mathbb{G}_{t_{k-1}}\right] \\
& =\left(u_{j}-m_{j}\left(t_{k-1}\right)\right)^{2} q^{2} m_{j}^{2(q-1)}\left(t_{k-1}\right)\left[\delta_{j, j}^{2}+\sum_{l=1, l \neq j}^{n} \delta_{j, l}^{2} m_{l}^{2}\left(t_{k-1}\right)\right] \Delta t_{k} \\
& +q^{2} m_{j}^{2 q}\left(t_{k}\right) M_{j}^{2}(s m)+q^{2} m_{j}^{2 q}\left(t_{k}\right) M_{j}^{2}(b m),
\end{aligned}
$$

and

$$
\begin{aligned}
& E\left[\Delta p_{j}^{q}\left(t_{k}\right) \mid \mathbb{G}_{t_{k-1}}\right]=q p_{j}^{q-1}\left(t_{k-1}\right) p_{j}\left(t_{k-1}\right)\left[\gamma_{j, j}\left(m_{j}\left(t^{-}\right)-p_{j}\left(t^{-}\right)\right)+\beta_{j}+\sum_{l=1}^{n} \gamma_{j, l} m_{l}(t)\right] \Delta t_{k} \\
& +\frac{1}{2} q(q-1) p_{j}^{q}\left(t_{k}\right)\left[\sigma_{j, j}^{2}+\sum_{l=1, l \neq j}^{n} \sigma_{j, l}^{2} p_{l}^{2}\left(t_{k-1}\right)\right]+q p_{j}^{q}\left(t_{k-1}\right) M_{j}^{1}(b p), \\
& E\left[\left[\Delta p_{j}^{q}\left(t_{k}\right)-E\left[\Delta p_{j}^{q}\left(t_{k}\right) \mid \mathbb{G}_{t_{k-1}}\right]\right]^{2} \mid \mathbb{G}_{t_{k-1}}\right]=q^{2} p_{j}^{2 q}\left(t_{k-1}\right)\left[\sigma_{j, j}^{2}+\sum_{l=1, l \neq j}^{n} \sigma_{j, l}^{2}\left(t_{k-1}\right) p_{j}^{2}\left(t_{k-1}\right)\right] \Delta t_{k} \\
& +q^{2} p_{j}^{2 q}\left(t_{k-1}\right) M_{j}^{2}(s p) \Delta t_{k}+q^{2} p_{j}^{2 q}\left(t_{k-1}\right) M_{j}^{2}(b p) \Delta t_{k} .
\end{aligned}
$$

We note that the particular case $q=1$ in (21) and (23) yields the following systems:

$$
\begin{aligned}
& \left.E\left[\Delta m_{j}\left(t_{k}\right) \mid \mathbb{G}_{t_{k-1}}\right]=\left(u_{j}-m_{j}\left(t_{k-1}\right)\right)\left[\sum_{l=1}^{n} \kappa_{j, l} m_{l}(t)\right)\right] \Delta t_{k} \\
& +m_{j}\left(t_{k-1}\right) M_{j}^{1}(b m), \\
& E\left[\left[\Delta m_{j}\left(t_{k}\right)-E\left[\Delta m_{j}\left(t_{k}\right) \mid \mathbb{G}_{t_{k-1}}\right]\right]^{2} \mid \mathbb{G}_{t_{k-1}}\right]=\left(u_{j}-m_{j}\left(t_{k-1}\right)\right)^{2} \sum_{l=1} \delta_{j, l}^{2} m_{l}^{2}\left(t_{k-1}\right) \Delta t_{k} \\
& +m_{j}^{2}\left(t_{k-1}\right)\left[M_{j}^{2}(s m) \Delta t_{k}+M_{j}^{2}(b m) \Delta t_{k}\right],
\end{aligned}
$$

and

$$
\begin{aligned}
& E\left[\Delta p_{j}\left(t_{k}\right) \mid \mathbb{G}_{t_{k-1}}\right]=p_{j}\left(t_{k-1}\right)\left[\gamma_{j, j}\left(m_{j}\left(t^{-}\right)-p_{j}\left(t^{-}\right)\right)+\beta_{j}+\sum_{l=1, l \neq j} \gamma_{j, l} p_{l}(t)\right] \Delta t_{k} \\
& +p_{j}\left(t_{k-1}\right) M_{j}^{2}(b p), \\
& E\left[\left[\Delta p_{j}\left(t_{k}\right)-E\left[\Delta p_{j}\left(t_{k}\right) \mid \mathbb{G}_{t_{k-1}}\right]\right]^{2} \mid \mathbb{G}_{t_{k-1}}\right]=p_{j}^{2}\left(t_{k-1}\right)\left[\left[\sum_{l=1}^{n} \sigma_{j, l}^{2} p_{l}^{2}\left(t_{k-1}\right)\right] \Delta t_{k}\right. \\
& \left.+M_{2}^{s}\left(p_{j}\right) \Delta t_{k-1}+M_{2}^{b}\left(p_{j}\right) \Delta t_{k-1}\right] .
\end{aligned}
$$

When $q=1$, from the second equation of the system in (31) we have:

$$
\begin{aligned}
& \left(u_{j}-m_{j}\left(t_{k-1}\right)\right)^{2}\left[\sum_{l=1} \delta_{j, l}^{2} m_{l}^{2}\left(t_{k-1}\right)\right]=E\left[\left[\Delta m_{j}\left(t_{k}\right)-E\left[\Delta m_{j}\left(t_{k}\right) \mid \mathbb{G}_{t_{k-1}}\right]\right]^{2} \mid \mathbb{G}_{t_{k-1}}\right] \\
& -m_{j}^{2}\left(t_{k}\right) M_{j}^{2}(s m)-m_{j}^{2}\left(t_{k}\right) M_{j}^{2}(b m) .
\end{aligned}
$$

Using (35) in the first moment equation of (27) yields:

$$
\begin{aligned}
& \left.E\left[\Delta m_{j}^{q}\left(t_{k}\right) \mid \mathbb{G}_{t_{k-1}}\right]=q m_{j}^{q-1}\left(t_{k-1}\right)\left(u_{j}-m_{j}\left(t_{k-1}\right)\right)\left[\sum_{l=1}^{n} \kappa_{j, l} m_{l}\left(t^{-}\right)\right)\right] \Delta t_{k} \\
& +\frac{1}{2} q(q-1) m_{j}^{q-2}\left(t_{k-1}\right)\left[E\left[\left[\Delta m_{j}\left(t_{k}\right)-E\left[\Delta m_{j}\left(t_{k}\right) \mid \mathbb{G}_{t_{k-1}}\right]\right]^{2} \mid \mathbb{G}_{t_{k-1}}\right]-m_{j}^{2}\left(t_{k}\right) M_{j}^{2}(s m)\right. \\
& \left.-m_{j}^{2}\left(t_{k}\right) M_{j}^{2}(b m)\right]+q m_{j}^{q}\left(t^{-}\right) M_{j}^{1}(b m),
\end{aligned}
$$


hence,

$$
\begin{aligned}
& E\left[\Delta m_{j}^{q}\left(t_{k}\right) \mid \mathbb{G}_{t_{k-1}}\right]-\frac{1}{2} q(q-1) m_{j}^{q-2}\left(t_{k-1}\right) E\left[\left[\Delta m_{j}\left(t_{k}\right)-E\left[\Delta m_{j}\left(t_{k}\right) \mid \mathbb{G}_{t_{k-1}}\right]\right]^{2} \mid \mathbb{G}_{t_{k-1}}\right] \\
& +\frac{1}{2} q(q-1) m_{j}^{q}\left(t_{k}\right) M_{j}^{2}(s m)+\frac{1}{2} q(q-1) m_{j}^{q}\left(t_{k}\right) M_{j}^{2}(b m)-q m_{j}^{q}\left(t^{-}\right) M_{j}^{1}(b m) \\
& =\kappa_{j j}\left[q u_{j} m_{j}^{q}\left(t_{k-1}\right)-q m_{j}^{q+1}\left(t_{k-1}\right)\right]+\kappa_{j l}\left[q u_{j} m_{j}^{q-1}\left(t_{k-1}\right) m_{l}\left(t_{k-1}\right)-q m_{j}^{q}\left(t_{k-1}\right) m_{l}\left(t_{k-1}\right)\right] .
\end{aligned}
$$

Writing the estimating equations at time $t_{k}$ yields:

$$
\begin{aligned}
& \sum_{k=s-n_{s}}^{s-1} E\left[\Delta m_{j}^{q}\left(t_{k}\right) \mid \mathbb{G}_{t_{k-1}}\right]-\frac{1}{2} q(q-1) \sum_{k=s-n_{s}}^{s-1} m_{j}^{q-2}\left(t_{k-1}\right) E\left[\left[\Delta m_{j}\left(t_{k}\right)-E\left[\Delta m_{j}\left(t_{k}\right) \mid \mathbb{G}_{t_{k-1}}\right]\right]^{2} \mid \mathbb{G}_{t_{k-1}}\right] \\
& +\frac{1}{2} q(q-1) M_{j}^{2}(s m) \sum_{k=s-n_{s}}^{s-1} m_{j}^{q}\left(t_{k}\right)+\frac{1}{2} q(q-1) M_{j}^{2}(b m) \sum_{k=s-n_{s}}^{s-1} m_{j}^{q}\left(t_{k}\right)-q \sum_{k=s-n_{s}}^{s-1} m_{j}^{q}\left(t^{-}\right) M_{j}^{1}(b m) \\
& =\kappa_{j j}\left[q u_{j} \sum_{k=s-n_{s}}^{s-1} m_{j}^{q}\left(t_{k-1}\right)-q \sum_{k=s-n_{s}}^{s-1} m_{j}^{q+1}\left(t_{k-1}\right)\right]+\kappa_{j l}\left[q u_{j} \sum_{k=s-n_{s}}^{s-1} m_{j}^{q-1}\left(t_{k-1}\right) m_{l}\left(t_{k-1}\right)\right. \\
& \left.-q \sum_{k=s-n_{s}}^{s-1} m_{j}^{q}\left(t_{k-1}\right) m_{l}\left(t_{k-1}\right)\right]
\end{aligned}
$$

Let us set:

$$
\begin{aligned}
& A_{j, m}^{q}=q \sum_{k=s-n_{s}}^{s-1} m_{j}^{q}\left(t_{k-1}\right)\left(u_{j}-m_{j}\left(t_{k-1}\right)\right)=a_{1}^{q} u+a_{2}^{q} \\
& B_{j, m}^{q}=q \sum_{k=s-n_{s}}^{s-1} m_{j}^{q-1}\left(t_{k-1}\right)\left(u_{j}-m_{j}\left(t_{k-1}\right)\right) m_{l}\left(t_{k-1}\right)=b_{1}^{q} u+b_{2}^{q} \\
& C_{j, m}^{q}=\sum_{k=s-n_{s}}^{s-1} E\left[\Delta m_{j}^{q}\left(t_{k}\right) \mid \mathbb{G}_{t_{k-1}}\right] \\
& -\frac{1}{2} q(q-1) \sum_{k=s-n_{s}}^{s-1} m_{j}^{q-2}\left(t_{k-1}\right) E\left[\left[\Delta m_{j}\left(t_{k}\right)-E\left[\Delta m_{j}\left(t_{k}\right) \mid \mathbb{G}_{t_{k-1}}\right]\right]^{2} \mid \mathbb{G}_{t_{k-1}}\right] \\
& +\frac{1}{2} q(q-1) M_{j}^{2}(s m) \sum_{k=s-n_{s}}^{s-1} m_{j}^{q}\left(t_{k}\right)+\frac{1}{2} q(q-1) M_{j}^{2}(b m) \sum_{k=s-n_{s}}^{s-1} m_{j}^{q}\left(t_{k}\right)-q \sum_{k=s-n_{s}}^{s-1} m_{j}^{q}\left(t^{-}\right) M_{j}^{1}(b m) \\
& a_{1}^{q}=q \sum_{k=s-n_{s}}^{s-1} m_{j}^{q}\left(t_{k-1}\right), a_{2}^{q}=q \sum_{k=s-n_{s}}^{s-1} m_{j}^{q+1}\left(t_{k-1}\right) \\
& b_{1}^{q}=q \sum_{k=s-n_{s}}^{s-1} m_{l}^{q-1}\left(t_{k-1}\right) m_{l}\left(t_{k-1}\right), b_{2}^{q}=q \sum_{k=s-n_{s}}^{s-1} m_{j}^{q}\left(t_{k-1}\right) m_{l}\left(t_{k-1}\right) .
\end{aligned}
$$

For $l \in\{1,2\}$ with, $l \neq j$ we seek to estimate the parameters $u_{j}, \kappa_{l j}^{m}$ and $\kappa_{l j}^{m}$. The deterministic interaction coefficient parameters $\kappa_{l j}$ are estimated using the first moment equation in (38), for any three real values $q_{1}, q_{2}$ and $q_{3}$ of the parameter $q$.

$$
\begin{aligned}
A_{j, m}^{q_{1}} \kappa_{j j}+B_{j, m}^{q_{1}} \kappa_{l j} & =C_{j, m}^{q_{1}} \\
A_{j, m}^{q_{2}} \kappa_{j j}+B_{j, m}^{q_{2}} \kappa_{l j} & =C_{j, m}^{q_{2}}, \\
A_{j, m}^{q_{3}} \kappa_{j j}+B_{j, m}^{q_{3}} \kappa_{l j} & =C_{j, m}^{q_{3}},
\end{aligned}
$$


yielding the solutions:

$$
\begin{aligned}
& u_{j}^{2}\left[a_{1}^{q_{3}} b_{1}^{q_{2}} c^{q_{1}}-a_{1}^{q_{3}} b_{1}^{q_{1}} c^{q_{2}}+a_{1}^{q_{1}} b_{1}^{q_{3}} c^{q_{2}}-a_{1}^{q_{2}} b_{1}^{q_{3}} c^{q_{1}}-a_{1}^{q_{1}} b_{1}^{q_{2}} c^{q_{3}}+a_{1}^{q_{2}} b_{1}^{q_{1}} c^{q_{3}}\right] \\
& +u_{j}\left[a_{1}^{q_{3}} b_{2}^{q_{2}} c^{q_{1}}+a_{2}^{q_{3}} b_{1}^{q_{2}} c^{q_{1}}-a_{1}^{q_{3}} b_{2}^{q_{2}} c^{q_{2}}-a_{2}^{q_{3}} b_{1}^{q_{1}} c^{q_{2}}+a_{1}^{q_{1}} b_{2}^{q_{3}} c^{q_{2}}+a_{2}^{q_{1}} b_{1}^{q_{3}} c^{q_{2}}-a_{2}^{q_{2}} b_{1}^{q_{3}} c^{q_{1}}-a_{1}^{q_{2}} b_{2}^{q_{3}} c^{q_{1}}\right. \\
& \left.\quad-a_{1}^{q_{1}} b_{2}^{q_{2}} c^{q_{3}}-a_{2}^{q_{1}} b_{1}^{q_{2}} c^{q_{3}}+a_{1}^{q_{2}} b_{2}^{q_{1}} c^{q_{1}}+a_{1}^{q_{1}} b_{2}^{q_{2}} c^{q_{3}}\right] \\
& +\left[a_{2}^{q_{3}} b_{2}^{q_{2}} c^{q_{1}}-a_{2}^{q_{3}} b_{2}^{q_{1}} c^{q_{2}}+a_{2}^{q_{1}} b_{2}^{q_{3}} c^{q_{2}}-a_{2}^{q_{2}} b_{2}^{q_{3}} c^{q_{1}}-a_{2}^{q_{1}} b_{2}^{q_{2}} c^{q_{3}}+a_{2}^{q_{2}} b_{2}^{q_{1}} c^{q_{3}}\right]=0 \\
& \kappa_{j j}=\frac{B_{j, m}^{q_{2}} C_{j, m}^{q_{1}}-C_{j, m}^{q_{2}} B_{j, m}^{q_{1}}}{B_{j, m}^{q_{2}} A_{j, m}^{q_{1}}-A_{j, m}^{q_{2}} B_{j, m}^{q_{1}}} \\
& \kappa_{l j}=\frac{C_{j, m}^{q_{2}} A_{j, m}^{q_{1}}-A_{j, m}^{q_{2}} C_{j, m}^{q_{1}}}{B_{j, m}^{q_{2}} A_{j, m}^{q_{1}}-A_{j, m}^{q_{2}} B_{j, m}^{q_{1}}} .
\end{aligned}
$$

We estimate $\delta_{j l}, l \in\{1,2\}, l \neq j$ the continuous random interaction coefficients of the mean processes $\left(m_{l}(t)\right)_{t \in[0, T]}, l \in\{1,2\}$ associated with the Brownian motion. We use the second order moment equation in (28)

$$
\begin{aligned}
& \sum_{k=s-n_{s}}^{s-1} E\left[\left[\Delta m_{j}^{q}\left(t_{k}\right)-E\left[\Delta m_{j}^{q}\left(t_{k}\right) \mid \mathbb{G}_{t_{k-1}}\right]\right]^{2} \mid \mathbb{G}_{t_{k-1}}\right]-q^{2} M_{j}^{2}(s m) \sum_{k=s-n_{s}}^{s-1} m_{j}^{2 q}\left(t_{k}\right) \\
& -q^{2} M_{j}^{2}(b m) \sum_{k=s-n_{s}}^{s-1} m_{j}^{2 q}\left(t_{k}\right) \\
& =\delta_{j j}^{2} \sum_{k=s-n_{s}}^{s-1}\left(u_{j}-m_{j}\left(t_{k-1}\right)\right)^{2} q^{2} m_{j}^{2(q-1)}\left(t_{k-1}\right)+\delta_{j l}^{2} \sum_{k=s-n_{s}}^{s-1}\left(u_{j}-m_{j}\left(t_{k-1}\right)\right)^{2} q^{2} m_{j}^{2(q-1)}\left(t_{k-1}\right) m_{l}^{2}\left(t_{k-1}\right)
\end{aligned}
$$

applied to any two distinct values of $q, q_{1}$ and $q_{2}$ as follows:

$$
\begin{aligned}
A_{j, m}^{q_{1}, j} \delta_{j j}^{2}+B_{j, m}^{q_{1}, l} \delta_{l j}^{2} & =C_{j, m}^{1} \\
A_{j, m}^{q_{2}, j} \delta_{j j}^{2}+B_{j, m}^{q_{2}, l} \delta_{l j}^{2} & =C_{j, m}^{2},
\end{aligned}
$$

where the coefficients are defined as follows:

$$
\begin{aligned}
& A_{j, m}^{q}=\sum_{k=s-n_{s}}^{s-1}\left(u_{j}-m_{j}\left(t_{k-1}\right)\right)^{2} q^{2} m_{j}^{2 q}\left(t_{k-1}\right) \Delta t_{k} \\
& B_{j, m}^{q}=\sum_{k=s-n_{s}}^{s-1}\left(u_{j}-m_{j}\left(t_{k-1}\right)\right)^{2} q^{2} m_{j}^{2(q-1)}\left(t_{k-1}\right) m_{l}^{2}\left(t_{k-1}\right) \Delta t_{k} \\
& C_{j, m}^{q}=\sum_{k=s-n_{s}}^{s-1} E\left[\left[\Delta m_{j}^{q}\left(t_{k}\right)-E\left[\Delta m_{j}^{q}\left(t_{k}\right) \mid \mathbb{G}_{t_{k-1}}\right]\right]^{2} \mid \mathbb{G}_{t_{k-1}}\right]-q^{2} M_{j}^{2}(s m) \sum_{k=s-n_{s}}^{s-1} m_{j}^{2 q}\left(t_{k}\right) \\
& -q^{2} M_{j}^{2}(b m) \sum_{k=s-n_{s}}^{s-1} m_{j}^{2 q}\left(t_{k}\right)
\end{aligned}
$$

this, therefore yields the solutions:

$$
\begin{gathered}
\delta_{j j}^{2}=\frac{C_{j, m}^{q_{1}, l} B_{j, m}^{q_{2}, l}-B_{j, m}^{q_{1}, l} C_{j, m}^{q_{2}, l}}{A_{j, m}^{q_{1}, j} B_{j, m}^{q_{2}, l}-A_{j, m}^{q_{2}, j} B_{j, m}^{q_{1}, l}} \\
\delta_{l j}^{2}=\frac{A_{j, m}^{q_{1}, j} C_{j, m}^{q_{2}, l}-C_{j, m}^{q_{1}, l} A_{j, m}^{q_{2}, j}}{A_{j, m}^{q_{2}, j} B_{j, m}^{q_{2}, l}-A_{j, m}^{q_{2}, j} B_{j, m}^{q_{1}, l}} .
\end{gathered}
$$


We turn our attention to estimating the parameters of the price processes $p_{j}, j \in\{1,2\}$. Equation of (30) has parameters $\sigma_{l j}^{2}, l \in\{1,2\}$. From

$$
\begin{aligned}
& \sum_{k=s-n_{s}}^{s-1} E\left[\left[\Delta p_{j}^{q}\left(t_{k}\right)-E\left[\Delta p_{j}^{q}\left(t_{k}\right) \mid \mathbb{G}_{t_{k-1}}\right]\right]^{2} \mid \mathbb{G}_{t_{k-1}}\right]-q^{2} \sum_{k=s-n_{s}}^{s-1} p_{j}^{2 q}\left(t_{k-1}\right) M_{j}^{2}(s p) \Delta t_{k} \\
& -q^{2} \sum_{k=s-n_{s}}^{s-1} p_{j}^{2 q}\left(t_{k-1}\right) M_{j}^{2}(b p) \Delta t_{k} \\
& =q^{2} \sum_{k=s-n_{s}}^{s-1} p_{j}^{2 q}\left(t_{k-1}\right)\left[\sigma_{j, j}^{2}\left(t_{k-1}\right)+\sum_{l=1, l \neq j}^{n} \sigma_{j, l}^{2}\left(t_{k-1}\right) p_{l}^{2}\left(t_{k-1}\right)\right] \Delta t_{k} .
\end{aligned}
$$

The parameters $\sigma_{l j}^{2}, l \in\{1,2\}$ are estimated through the general equation,

$$
E_{q, j} \sigma_{j j}^{2}+F_{q, l} \sigma_{l j}^{2}=D_{q, l}
$$

where,

$$
\begin{aligned}
E_{q, j} & =q^{2} \sum_{k=s-n_{s}}^{s-1} p_{j}^{2 q}\left(t_{k-1}\right) \\
F_{q, l} & =q^{2} \sum_{k=s-n_{s}}^{s-1} p_{j}^{2 q}\left(t_{k-1}\right) p_{l}^{2}\left(t_{k-1}\right) \\
D_{q, l} & =\sum_{k=s-n_{s}}^{s-1} E\left[\left[\Delta p_{j}^{q}\left(t_{k}\right)-E\left[\Delta p_{j}^{q}\left(t_{k}\right) \mid \mathbb{G}_{t_{k-1}}\right]\right]^{2} \mid \mathbb{G}_{t_{k-1}}\right]-q^{2} \sum_{k=s-n_{s}}^{s-1} p_{j}^{2 q}\left(t_{k-1}\right) M_{j}^{2}(s p) \Delta t_{k} \\
-q^{2} & \sum_{k=s-n_{s}}^{s-1} p_{j}^{2 q}\left(t_{k-1}\right) M_{j}^{2}(b p) \Delta t_{k}
\end{aligned}
$$

For two distinct values $q_{1}$ and $q_{2}$ of $q$ we form the following system:

$$
\begin{aligned}
& E_{q_{1}, j} \sigma_{j j}^{2}+F_{q_{1}, l} \sigma_{l j}^{2}=D_{q_{1}, l} \\
& E_{q_{2}, j} \sigma_{j j}^{2}+F_{q_{2}, l} \sigma_{l j}^{2}=D_{q_{2}, l},
\end{aligned}
$$

with solutions;

$$
\begin{aligned}
\sigma_{j j}^{2} & =\frac{F_{q_{2}, l} D_{q_{1}, l}-D_{q_{2}, l} F_{q_{1}, l}}{E_{q_{1}, j} F_{q_{2}, l}-E_{q_{2}, j} F_{q_{1}, l}}, \\
\sigma_{l j}^{2} & =-\frac{D_{q_{2}, l} E_{q_{1}, j}-D_{q_{1}, l} E_{q_{2}, j}}{E_{q_{1}, j} F_{q_{2}, l}-E_{q_{2}, j} F_{q_{1}, l}} .
\end{aligned}
$$

The last parameters of the price processes are the deterministic interaction coefficient parameters $\gamma_{j j}, \beta_{j}$ and $\gamma_{l j}, l \neq j$. In order to estimate them, we assume the parameters $\sigma_{j l}, l \in\{1,2\}$ known from the estimation equations (48). We use the first moment equation in (29).

$$
\begin{aligned}
& \sum_{k=s-n_{s}}^{s-1} E\left[\Delta p_{j}^{q}\left(t_{k}\right) \mid \mathbb{G}_{t_{k-1}}\right]-\frac{1}{2} q(q-1) \sum_{k=s-n_{s}}^{s-1} p_{j}^{q}\left(t_{k}\right)\left[\sigma_{j, l}^{2}+\sigma_{j, l}^{2} p_{l}^{2}\left(t_{k-1}\right)\right]-q \sum_{k=s-n_{s}}^{s-1} p_{j}^{q}\left(t_{k-1}\right) M_{j}^{1}(b p) \\
& =q \sum_{k=s-n_{s}}^{s-1} p_{j}^{q}\left(t_{k}\right)\left[\gamma_{j j}\left(m_{j}\left(t_{k-1}\right)-p_{j}\left(t_{k-1}\right)\right)+\beta_{j}+\sum_{l=1, l \neq j}^{n} \gamma_{j l} p_{l}\left(t_{k-1}\right)\right] \Delta t_{k}
\end{aligned}
$$

Equation (50) could also be written

$$
K_{q, j} \gamma_{j j}+Q_{q} \beta_{j}+O_{q, l} \gamma_{l j}=S_{q, l}
$$


where the equation coefficients are expressed as follows,

$$
\begin{aligned}
& S_{q, l}=\sum_{k=s-n_{s}}^{s-1} E\left[\Delta p_{j}^{q}\left(t_{k}\right) \mid \mathbb{G}_{t_{k-1}}\right]-\frac{1}{2} q(q-1) \sum_{k=s-n_{s}}^{s-1} p_{j}^{q}\left(t_{k}\right)\left[\sum_{l=1}^{n} \sigma_{j, l}^{2} p_{l}^{2}\left(t_{k-1}\right)\right] \\
& -q \sum_{k=s-n_{s}}^{s-1} p_{j}^{q}\left(t_{k-1}\right) M_{j}^{1}(b p) \\
& O_{q, l}=q \sum_{k=s-n_{s}}^{s-1} p_{j}^{q}\left(t_{k}\right) \\
& Q_{q}=q \sum_{k=s-n_{s}}^{s-1} p_{j}^{q}\left(t_{k}\right) \\
& K_{q, j}=q \sum_{k=s-n_{s}}^{s-1} p_{j}^{q}\left(t_{k}\right)\left(m_{j}\left(t_{k-1}\right)-p_{j}\left(t_{k-1}\right)\right)
\end{aligned}
$$

For three distinct values $q_{1}, q_{2}$ and $q_{3}$ of $q$, we form the system:

$$
\begin{aligned}
& K_{q_{1}, j} \gamma_{j j}+Q_{q_{1}} \beta_{j}+O_{q_{1}, l} \gamma_{l j}=S_{q_{1}, l}, \\
& K_{q_{2}, j} \gamma_{j j}+Q_{q_{2}} \beta_{j}+O q_{2}, l \gamma_{l j}=S_{q_{2}, l}, \\
& K_{q_{3}, j} \gamma_{j j}+Q_{q_{3}} \beta_{j}+O_{q_{3}, l} \gamma_{l j}=S_{q_{3}, l} .
\end{aligned}
$$

Solutions are expressed in closed form through Cramer rule

$$
\gamma_{j j}=\frac{\left|\begin{array}{lll}
S_{q_{1}, l} & Q_{q_{1}} & O_{q_{1}, l} \\
S_{q_{2}, l} & Q_{q_{2}} & O_{q_{2}, l} \\
S_{q_{3}, l} & Q_{q_{3}} & O_{q_{3}, l}
\end{array}\right|}{\left|\begin{array}{lll}
K_{q_{1}, j} & Q_{q_{1}} & O_{q_{1}, l} \\
K_{q_{2}, j} & Q_{q_{2}} & O_{q_{2}, l} \\
K_{q_{3}, j} & Q_{q_{3}} & O_{q_{3}, l}
\end{array}\right|}, \beta_{j}=\frac{\left|\begin{array}{ccc}
K_{q_{1}, j} & S_{q_{1}, l} & O_{q_{1}, l} \\
K_{q_{2}, j} & S_{q_{2}, l} & O_{q_{2}, l} \\
K_{q_{3}, j} & S_{q_{3}, l} & O_{q_{3}, l}
\end{array}\right|}{\left|\begin{array}{cccc}
K_{q_{1}, j} & Q_{q_{1}} & O_{q_{1}, l} \\
K_{q_{2}, j} & Q_{q_{2}} & O_{q_{2}, l} \\
K_{q_{3}, j} & Q_{q_{3}} & O_{q_{3}, l}
\end{array}\right|}, \gamma_{l j}=\frac{\left|\begin{array}{ccc}
K_{q_{1}, j} & Q_{q_{1}} & S_{q_{1}, l} \\
K_{q_{2}, j} & Q_{q_{2}} & S_{q_{2}, l} \\
K_{q_{3}, j} & Q_{q_{3}} & S_{q_{3}, l}
\end{array}\right|}{\left|\begin{array}{llll}
K_{q_{1}, j} & Q_{q_{1}} & O_{q_{1}, l} \\
K_{q_{2}, j} & Q_{q_{2}} & O_{q_{2}, l} \\
K_{q_{3}, j} & Q_{q_{3}} & O_{q_{3}, l}
\end{array}\right|} .
$$

\subsection{Modeling of Asset Prices with a Regime Switching Heston Model}

This illustration extends Elliott et al. (2015) and presents how vanilla option pricing could be performed using a model with stochastic volatility in a market with semi Markov regimes. However, the results and proofs in this second illustration of the general model of coupled stochastic dynamics will rely on a different but equivalent notation for market regimes. We first introduce the new notation for the semi Markov process and a few necessary results.

Definition 3.1. 1. The semi Markov process $\left(\theta_{t}\right)_{t \in[0, T]}$ is now represented by a vector process denoted $\left(\Theta_{t}\right)_{t \in[0, T]}$ with $\Theta_{t}=\left(1_{\left(\theta_{t}=1\right)}, 1_{\left(\theta_{t}=2\right)}, \ldots, 1_{\left(\theta_{t}=m\right)}\right)$,

2. $\Lambda\left(y_{t}\right)=\left(\lambda_{i j}\left(y_{t}\right)\right)_{i, j \leq m}$ denotes the conditional matrix of intensities of the semi Markov process $\left(\Theta_{t}\right)_{t \in[0, T]}$,

3. the notation $\Lambda^{T}$ denotes the matrix transposed of $\Lambda$,

4. we denote the states of the semi Markov process $\left(\Theta_{t}\right)_{t \in[0, T]}, e_{1}, \ldots e_{m}$, where $e_{i}=\underbrace{(0, \ldots 0,1,0, \ldots 0)}_{i-t h}$ and $\mathbf{I}$ represents the identity matrix.

We establish a martingale theorem for the vector valued semi Markov process as a particular case of Brémaud (1981).

Lemma 3.1. Let $\Theta_{t}=\left(1_{\left(\theta_{t}=1\right)}, 1_{\left(\theta_{t}=2\right)}, \ldots, 1_{\left(\theta_{t}=m\right)}\right)$ and $\Lambda(t)=\left(\lambda_{i j}\left(y_{t}\right)\right)_{i, j \leq m}$ as in Definition 3.1, be the vector carrying instantaneous states of the semi Markov process $\Theta_{t}$ and the conditional intensity matrix of $\Theta_{t}$. There exists an $\mathbb{R}^{m}$ vector valued martingale process $M_{t}$, right continuous with left limits such that:

$$
\Theta_{t}=\Theta_{0}+\int_{t_{0}}^{t} \Lambda^{\prime}\left(y_{u^{-}}\right) \Theta_{u}^{-} d u+M_{t}
$$


Proof. From Definition 3.1 and the intensity theorem for general marked point processes in Brémaud (1981), the conditional intensity process $\lambda_{i}$ of $1_{\left(\theta_{t}=i\right)}$ could be expressed as follows:

$$
\begin{aligned}
\lambda_{i}(t) & =\sum_{n \geq 0} p_{\theta_{n} i} \frac{f_{\theta_{n} i}\left(t-T_{n}\right) d t}{S_{\Theta_{n}}\left(t-T_{n}\right)} 1_{\left(T_{n} \leq t<T_{n+1}\right)} 1_{\left(T_{n}<\infty\right)} \\
& =\sum_{j \in E} \sum_{n \geq 0} p_{j i} \frac{f_{j i}\left(t-T_{n}\right) d t}{S_{\Theta_{n}}\left(t-T_{n}\right)} 1_{T_{n} \leq t<T_{n+1}} 1_{T_{n}<\infty} \\
& =\sum_{j \in E} \lambda_{j, i}\left(t-T_{n}\right) 1_{\theta_{t}=j}, \text { where, } \lambda_{j, i}=\sum_{n \geq 0} p_{j i} \frac{f_{j i}\left(t-T_{n}\right) d t}{S_{\Theta_{n}}\left(t-T_{n}\right)} 1_{T_{n} \leq t<T_{n+1}} 1_{T_{n}<\infty} .
\end{aligned}
$$

Hence from Brémaud (1981), there exists real valued cádlág martingale processes $M^{i}(t), \forall i=1, \ldots, m$ such that

$$
M^{i}(t)=1_{\left(\theta_{t}=i\right)}-\int_{0}^{t} \lambda_{i}\left(y_{s^{-}}\right) d s
$$

We set $M(t)=\left(M^{1}(t), \ldots, M^{m}(t)\right)$ and rewrite

$$
\begin{aligned}
d\left(1_{\left(\theta_{t}=i\right)}\right) & =\sum_{j \in E} \lambda_{j, i}\left(y_{t^{-}}\right) 1_{\left(\theta_{t^{-}}=j\right)} d t+d M^{i}(t) \\
d\left(1_{\left(\theta_{t}=i\right)}\right) & =\left(\lambda_{1, i}\left(y_{t^{-}}\right), \lambda_{2, i}\left(y_{t^{-}}\right), \ldots, \lambda_{m, i}\left(y_{t^{-}}\right)\right) . \Theta_{t}^{-} d t+d M^{i}(t) \\
& \text { hence: } \\
d \Theta_{t} & =\Lambda\left(y_{t^{-}}\right)^{\prime} \Theta_{t}^{-} d t+d M(t),
\end{aligned}
$$

hence proving a martingale decomposition of the vector process $\left(\Theta_{t}\right)_{t \in[0, T]}$.

We consider the particular case where the asset price and its volatility follow the risk neutral Heston Model:

$$
\begin{aligned}
& d x_{t}=\left(r\left(t, \Theta_{t}, y_{t}\right)-.5 \sigma\left(t, \Theta_{t}, y_{t}\right)\right) d t+x_{t} \sqrt{\sigma\left(t, \Theta_{t}, y_{t}\right)} d W_{t}^{1} \\
& d \sigma\left(t, \Theta_{t}, y_{t}\right)=a\left(t, \Theta_{t}, y_{t}\right)\left(b\left(t, \Theta_{t}, y_{t}\right)-\sigma\left(t, \Theta_{t}, y_{t}\right)\right) d t+v\left(t, \Theta_{t}, y_{t}\right) \sqrt{\sigma\left(t, \Theta_{t}, y_{t}\right)} d W_{t}^{2} \\
& \text { with: } d W_{t}^{1} d W_{t}^{2}=\rho_{t} d t, \text { and } \sigma\left(0, \Theta_{0}, y_{0}\right)=\sigma_{0}>0,
\end{aligned}
$$

where $r$ is the risk free interest rate, $x$ is the log asset price model, $\sigma$ is the asset volatility, $a$ is the speed of the mean reversion of the asset volatility, $b$ is the long term asset volatility and $v$ determines the variance of the volatility which is referred to as the volatility of volatility.

We make the following simplifying assumptions on the model parameters:

1. A sufficient condition for the volatility remains non negative we assume: $2 a\left(t, \Theta_{t}, y_{t}\right) b\left(t, \theta_{t}, y_{t}\right)>v^{2}\left(t, \Theta_{t}, y_{t}\right), \forall t \in$ $[0, T]$,

2. The only parameter subjected to mean reversion is the long term volatility: $r\left(t, \Theta_{t}, y_{t}\right)=r(t), \rho\left(t, \Theta_{t}, y_{t}\right)=\rho(t)$, $v\left(t, \Theta_{t}, y_{t}\right)=v(t)$

3. the speed of mean reversion of the volatility, the interest rate and the volatility of volatility are assumed to be positive and constant: $a(t)=a>0, r(t)=r>0, v(t)=v>0$.

4. The correlation between asset price and volatility is assume to be constant: $\rho(t)=\rho$.

The main goal is to derive a general formula for the vanilla European call option prices both from the perspective of Carr and Madan's algorithm and as an extension of the formula derived in Elliott et al. (2015). Both problems are simplified if one can derive an expression for the following conditional expectation:

$$
E\left(e^{i w x_{u}} \mid \mathbb{F}_{t}\right) \text {, for a fixed } u \geq t .
$$

We first present a useful Lemma providing a general conditional expectation formula needed in this illustration. 
Lemma 3.2. We denote $\boldsymbol{I}, \mathbf{1}=\underbrace{(1, \ldots, 1)}_{m}, f, \Lambda(s)=\left(\lambda_{i, j}(s)\right)_{1 \leq i, j \leq m}$ and $\Phi$ the $m \times m$ identity matrix, an $m \times 1$ vector of real numbers, a real valued process continuous in its second and third variables, the $m \times m$ real valued conditional intensity matrix of the semi Markov process $\Theta$ and the $m \times m$ matrix of real numbers satisfying the linear matrix partial differential equation with terminal condition:

$$
\frac{\partial \Phi}{\partial t}+\frac{\partial \Phi}{\partial y}+\boldsymbol{A}\left(t, y_{t}\right) \Phi\left(T, t, y_{t}\right)=0, \text { with } \Phi\left(T, T, y_{T}\right)=\boldsymbol{I}
$$

where:

$$
\begin{aligned}
& \boldsymbol{A}\left(T, t, y_{t}\right)=\Lambda^{\prime}\left(y_{t}\right)-\operatorname{diag}\left(\boldsymbol{f}\left(t, y_{t}\right)\right), \\
& \boldsymbol{f}(t, y)=\left(f\left(t, y_{t}, e_{1}\right), f\left(t, y_{t}, e_{2}\right), \ldots, f\left(t, y_{t}, e_{m}\right)\right) .
\end{aligned}
$$

We assume that the following conditions are satisfied:

$$
\begin{aligned}
& E\left[e^{-\int_{0}^{T} f\left(u, y_{u^{-}}, \Theta_{u^{-}}\right) d u} \mid \mathbb{H}_{t} \vee \mathbb{L}_{T}\right]<\infty, \forall t \in[0, T] \\
& \int_{0}^{T} \lambda_{i, j}(s) d s<\infty
\end{aligned}
$$

We define a real valued function $F$ and a vector valued function $\boldsymbol{F}$ as follows:

$$
\begin{aligned}
F\left(T, t, y_{t}, \Theta_{t}\right) & =E\left(e^{-\int_{t}^{T} f\left(u, y_{u^{-}}, \Theta_{u^{-}}\right) d u} \mid \mathbb{H}_{t} \vee \mathbb{L}_{T}\right), \\
\boldsymbol{F}\left(T, t, y_{t}\right) & =\left(F\left(T, t, y_{t}, e_{1}\right), F\left(T, t, y_{t}, e_{2}\right), \ldots, F\left(T, t, y_{t}, e_{m}\right)\right), .
\end{aligned}
$$

Let $K$ be a $m \times m$ real matrix function. $M$ is said to satisfy the bracket condition if $\forall t_{1}, t_{1} \in \mathbb{R}^{+},\left[K\left(t_{1}\right), K\left(t_{2}\right)\right]=0$, where [, ] denotes Lie matrix bracket.

1. The vector valued function $\boldsymbol{F}$ and the real valued function $F$ can be respectively expressed as follows:

$$
\begin{aligned}
\boldsymbol{F}\left(T, t, y_{t}\right) & =\Phi\left(T, t, y_{t}\right) \mathbf{1}, \\
F\left(T, t, y_{t}, \Theta_{t}\right) & =\left\langle\Phi\left(T, t, y_{t}\right) \mathbf{1}, \Theta_{t}\right\rangle=\left\langle\Phi\left(T, t, y_{t}\right) \Theta_{t}, \mathbf{1}\right\rangle,
\end{aligned}
$$

2. If $\boldsymbol{A}$ satisfies the bracket condition, a closed form expression for the fundamental matrix of (55) could be expressed as follows:

$$
\Phi\left(T, t, y_{t}\right)=\exp \left[\int_{y}^{T-t+y} \boldsymbol{A}(v-y+t, v) d v\right],
$$

Proof. We define the following product of processes,

$$
\begin{aligned}
P_{t} & =e^{-\int_{0}^{t} f\left(u, y_{u^{-}}, \Theta_{u^{-}}\right) d u} F\left(T, t, y_{t}, \theta_{t}\right) \\
& =e^{-\int_{0}^{t} f\left(u, y_{u^{-}}, \Theta_{u^{-}}\right) d u} E\left[e^{-\int_{t}^{T} f\left(u, y_{u^{-}}, \Theta_{u^{-}}\right) d u} \mid \mathbb{H}_{t} \vee \mathbb{L}_{T}\right] \\
& =E\left[e^{-\int_{0}^{T} f\left(u, y_{u^{-}}, \Theta_{u^{-}}\right) d u} \mid \mathbb{H}_{t} \vee \mathbb{L}_{T}\right] .
\end{aligned}
$$

In addition to being integrable as per assumption (56), the process $\left((P)_{t \in[0, T]}\right)$ satisfies the following properties:

$$
\begin{aligned}
E\left[P_{t} \mid \mathbb{H}_{s} \vee \mathbb{L}_{T}\right] & =E\left[E\left[e^{-\int_{0}^{T} f\left(u, y_{u^{-}}, \Theta_{u^{-}}\right) d u} \mid \mathbb{H}_{t} \vee \mathbb{L}_{T}\right] \mid \mathbb{H}_{s} \vee \mathbb{L}_{T}\right] \\
& =E\left[e^{-\int_{0}^{T} f\left(u, y_{u^{-}}, \Theta_{u^{-}}\right) d u} \mid \mathbb{H}_{s} \vee \mathbb{L}_{T}\right] \\
& =P_{s}, \forall s<t \text { with } s, t \in \mathbb{R} .
\end{aligned}
$$

Hence, $P_{t}$ has the martingale property with respect to the filtration $\left(\mathbb{H}_{t} \vee \mathbb{L}_{T}\right)_{t \in[0, T]}$. Writing Itó differential formula for $P_{t}$ from Applebaum (2009) yields:

$$
\begin{aligned}
& d\left[e^{-\int_{0}^{t} f\left(u, y_{u^{-}}, \Theta_{u^{-}}\right) d u} F\left(T, t, y_{t}, \Theta_{t}\right)\right]=-f\left(t^{-}, y_{t^{-}}, \Theta_{t^{-}}\right) e^{\int_{0}^{t} f\left(u, y_{u^{-}}, \Theta_{u^{-}}\right) d u} F\left(T, t, y_{t}, \Theta_{t}\right) d t \\
& +e^{-\int_{0}^{t} f\left(u, y_{u^{-}}, \Theta_{u^{-}}\right) d u} d F\left(T, t, y_{t}, \Theta_{t}\right) d t \\
& -f\left(t^{-}, \Theta t^{-}, y_{t^{-}}\right) e^{-\int_{0}^{t} f\left(u, \Theta_{u^{-}}\right) d u} F\left(T, t, y_{t}, \Theta_{t}\right) d t \\
& =-f\left(t^{-}, y_{t^{-}}, \Theta_{t^{-}}\right) e^{-\int_{0}^{t} f\left(u, \Theta_{u^{-}}\right) d u} F\left(T, t, y_{t}, \Theta_{t}\right) d t+e^{-\int_{0}^{t} f\left(u, \Theta_{u^{-}}\right) d u} d F\left(T, t, y_{t}, \Theta_{t}\right)
\end{aligned}
$$


Applying a particular case of Itó rule for semi Markov regime switching processes developed in Assonken and G. Ladde (2015) yields:

$$
d F\left(T, t, y_{t}, \theta_{t}\right)=\frac{\partial F}{\partial t} d t+\frac{\partial V}{\partial y} d t+\left\langle\boldsymbol{F}\left(T, s, y_{s^{-}}\right), d \Theta_{s}\right\rangle
$$

Hence,

$$
\begin{aligned}
& d\left[e^{-\int_{0}^{t} f\left(u, \Theta_{u^{-}}\right) d u} F\left(T, t, y_{t}, \Theta_{t}\right)\right]=-f\left(t^{-}, \Theta_{t^{-}}, y_{t^{-}}\right) e^{-\int_{0}^{t} f\left(u, \Theta_{u^{-}}\right) d u} F\left(T, t, y_{t}, \Theta_{t}\right) d t \\
& +e^{\int_{0}^{t} f\left(u, \Theta_{u^{-}}\right) d u}\left[\frac{\partial F}{\partial t} d t+\frac{\partial F}{\partial y} d t+\left\langle\boldsymbol{F}\left(T, t, y_{t^{-}}\right), d \Theta_{s}\right\rangle\right] \\
& =-f\left(t^{-}, \Theta_{t^{-}}, y_{t^{-}}\right) e^{-\int_{0}^{t} f\left(u, \Theta_{u^{-}}\right) d u} F\left(T, t, y_{t}, \Theta_{t}\right) d t \\
& +e^{-\int_{0}^{t} f\left(u, \Theta_{u^{-}}\right) d u}\left[\frac{\partial F}{\partial t}+\frac{\partial F}{\partial y}+\left\langle\boldsymbol{F}\left(T, t, y_{t^{-}}\right), \Lambda\left(y_{t^{-}}\right)^{T} \Theta_{t^{-}}\right\rangle\right] d t \\
& \left.+e^{-\int_{0}^{t} f\left(u, \Theta_{u^{-}}\right) d u}\left\langle\boldsymbol{F}\left(T, t, y_{t^{-}}\right), d M_{t^{-}}\right)\right\rangle \\
& =-e^{-\int_{0}^{t} f\left(u, \Theta_{u^{-}}\right) d u}\left\langle\operatorname{diag}\left(\boldsymbol{f}\left(t^{-}, y_{t^{-}}\right)\right) \boldsymbol{F}\left(T, t, y_{t}\right), \Theta_{t^{-}}\right\rangle d t \\
& +e^{-\int_{0}^{t} f\left(u, \Theta_{u^{-}}\right) d u}\left[\left\langle\frac{\partial \boldsymbol{F}}{\partial t}, \Theta_{t^{-}}\right\rangle d t+\left\langle\frac{\partial \boldsymbol{F}}{\partial y}, \Theta_{t^{-}}\right\rangle d t+\left\langle\Lambda\left(y_{t^{-}}\right) \boldsymbol{F}\left(T, t, y_{t^{-}}\right), \Theta_{t^{-}}\right\rangle\right] d t \\
& \left.+e^{-\int_{0}^{t} f\left(u, \Theta_{u^{-}}\right) d u}\left\langle\boldsymbol{F}\left(T, t, y_{t^{-}}\right), d M_{t^{-}}\right)\right\rangle .
\end{aligned}
$$

As $e^{\int_{0}^{t} f\left(u, y_{u^{-}}, \Theta_{u^{-}}\right) d u} F\left(T, t, y_{t}, \Theta_{t}\right)$ is a martingale process, the bounded variation term of Itó formula in (66) is identically zero. It reads:

$$
\frac{\partial \boldsymbol{F}}{\partial t}+\frac{\partial \boldsymbol{F}}{\partial y}+\boldsymbol{A}\left(t^{-}, y_{t^{-}}\right) \boldsymbol{F}\left(T, t, y_{t}\right)=0, \text { with } \boldsymbol{A}\left(t, y_{t}\right)=-\operatorname{diag}\left(\boldsymbol{f}\left(t, y_{t}\right)\right)+\Lambda^{\prime}\left(y_{t}\right) .
$$

Assuming matrix $\boldsymbol{A}$ has continuous components with respect to both $t$ and $y$ there exists $\Phi$ a fundamental solution of (69), ie $\Phi$ satisfies the matrix ODE

$$
\frac{\partial \Phi}{\partial t}+\frac{\partial \Phi}{\partial y}+\boldsymbol{A}\left(t^{-}, y_{t^{-}}\right) \Phi\left(T, t, y_{t}\right)=0, \text { with } \boldsymbol{A}\left(t, y_{t}\right)=-\operatorname{diag}\left(\boldsymbol{f}\left(t, y_{t}\right)\right)+\Lambda^{\prime}\left(y_{t}\right)
$$

Hence, the solution of the ODE (69) with terminal condition $\boldsymbol{F}\left(T, T, y_{T}\right)=\mathbf{1}$ is:

$$
\boldsymbol{F}\left(T, t, y_{t}\right)=\Phi\left(T, t, y_{t}\right) \mathbf{1}
$$

where $\Phi$ is solution of the matrix partial differential equation (70), with terminal condition $\Phi\left(T, T, y_{T}\right)=I$. This therefore proves (60). We establish (61) as follows:

$$
\begin{aligned}
& F\left(T, t, y_{t}, \Theta_{t}\right)=E\left(e^{\int_{t}^{T} f\left(u, y_{u^{-}}, \Theta_{u^{-}}\right) d u} \mid \mathbb{H}_{t} \vee \mathbb{L}_{T}\right) \\
& =\left\langle\boldsymbol{F}\left(T, t, y_{t}\right), \Theta_{t}\right\rangle, \text { From }(59) \\
& =\left\langle\Phi\left(T, t, y_{t}\right) \mathbf{1}, \Theta_{t}\right\rangle \text { Since the } e_{j}, j=1, \ldots, m \text { form an orthonormal basis } \\
& =\left\langle\Phi\left(T, t, y_{t}\right) \Theta_{t}, \mathbf{1}\right\rangle \text {. }
\end{aligned}
$$

The proof of part (2) proceeds from the result in part (1). Indeed, assuming the bracket condition is satisfied one can derive a closed form expression for the solution of (69). We first use the method of characteristic to solve the system of PDEs (69). We consider the variable transforms:

$$
\eta=t-y \text { and } \zeta=t+y
$$

Based on (71), we define the transforms $\tilde{\boldsymbol{F}}$ and $\tilde{\boldsymbol{A}}$ from $\boldsymbol{F}$ and $\boldsymbol{A}$, respectively, as functions of $(\eta, \zeta)$ :

$$
\left\{\begin{array}{l}
\tilde{\boldsymbol{F}}(T, \eta, \zeta)=\boldsymbol{F}\left(T, \frac{\eta+\zeta}{2}, \frac{-\eta+\zeta}{2}\right) \\
\tilde{\boldsymbol{A}}(\eta, \zeta)=\boldsymbol{A}\left(\frac{\eta+\zeta}{2}, \frac{-\eta+\zeta}{2}\right)
\end{array}\right.
$$

Simple algebra shows that the system of PDEs (69) becomes :

$$
\frac{\partial \tilde{\boldsymbol{F}}(T, \eta, \zeta)}{\partial \zeta}=-\frac{1}{2} \tilde{\boldsymbol{A}}(\eta, \zeta) \tilde{\boldsymbol{F}}(T, \eta, \zeta)
$$


Assuming continuity of the components of the matrix $\tilde{A}$, the ODE (73) has general solution

$$
\tilde{\boldsymbol{F}}(T, \eta, \zeta)=\exp \left[-\frac{1}{2} \int_{0}^{\zeta} \tilde{\boldsymbol{A}}(\eta, s) d s\right] . c(\eta)
$$

where $c$ is a vector function of $\eta$ only. Assuming the terminal condition $\boldsymbol{F}(T, T, y)=\mathbf{1}$, the function $c$ becomes

$$
\begin{aligned}
c(T-y) & =\exp \left[\frac{1}{2} \int_{0}^{T+y} \tilde{A}(T-y, s) d s\right], \\
& \text { which leads to }, \\
c(\eta) & =\exp \left[\frac{1}{2} \int_{0}^{2 T-\eta} \tilde{A}(\eta, s) d s\right] .
\end{aligned}
$$

Hence, the solution of the system of PDEs (69) becomes

$$
\begin{aligned}
\boldsymbol{F}(T, t, y) & =\exp \left[\frac{1}{2} \int_{t+y}^{2 T-t+y} \boldsymbol{A}\left(T, \frac{t-y+s}{2}, \frac{y-t+s}{2}\right) d s\right] . \mathbf{1} \\
& =\exp \left[\int_{y}^{T-t+y} \boldsymbol{A}(v-y+t, v) d v\right] . \mathbf{1},
\end{aligned}
$$

where $v=\frac{y-t+s}{2}$. Hence, one can verify that the fundamental matrix of the matrix system is:

$$
\Phi\left(T, t, y_{t}\right)=\exp \left[\int_{y}^{T-t+y} \boldsymbol{A}(v-y+t, v) d v\right] .
$$

We note that the semi Markov process $\theta_{t}$ paired with its corresponding backward recurrence time $y_{t}$ form a Markov process. This important fact allows to claim that the preceding conditional characteristic function is a function of only the current values of the variables. Next Lemma review the characteristic function formula of the log price in the context of Heston model with no market regime.

Lemma 3.3. If a log asset price $\left(x_{t}\right)_{t \in[0, T]}$ and its volatility process $\left(\sigma_{t}\right)_{t \in[0, T]}$ follow the dynamic of the model in (52) and (53), with one single market regime (that is no regime change), the characteristic function of the log price is expressed as follows:

$$
\begin{aligned}
E\left(e^{i w x_{u}} \mid \mathbb{F}_{t}\right) & =e^{A_{t}+B_{t} \sigma_{t}+i w x_{t}}, \forall u \geq t \\
A_{t} & =i r w(u-t)+a \int_{t}^{u} B_{s} b_{s} d s \\
B_{t} & =\frac{a-i \rho v w+\eta}{v^{2}}\left(\frac{1-e^{\eta(u-t)}}{1-\gamma e^{\eta(u-t)}}\right) \\
& \text { where, } \\
\eta & =\sqrt{(a-i \rho v w+\eta)^{2}+v^{2} w(w+i)} \\
\gamma & =\frac{a-i \rho v w+\eta}{a-i \rho v w+\eta}
\end{aligned}
$$

where, $i=\sqrt{-1}$.

Proof. The Markov property of the pair $\left(\Theta_{t}, y_{t}\right)$ implies that the quadruplet $\left(\Theta_{t}, y_{t}, x_{t}, \sigma_{t}\right)$ is Markovian as well. Therefore, we can use the notation:

$$
h\left(u, \theta_{t}, y_{t}, \sigma_{t}, x_{t}\right)=E\left(e^{i b x_{u}} \mid \mathbb{F}_{t}\right) \text {, for a fixed } u>t .
$$

We derive the system of partial differential equations satisfied by $h$ when the market has one single state $\theta_{t}=j, \forall t \in[0, T]$. From 9 Itó Lemma applied to $h$ yields:

$$
\begin{aligned}
d h\left(t, j, \sigma_{t}, x_{t}\right) & =\frac{\partial h\left(t, j, \sigma_{t}, x_{t}\right)}{\partial t} d t+\frac{\partial h\left(t, j, \sigma_{t}, x_{t}\right)}{\partial x} d x_{t}+\frac{\partial h\left(t, j, \sigma_{t}, x_{t}\right)}{\partial \sigma} d \sigma_{t} \\
& +\frac{1}{2} \frac{\partial^{2} h\left(t, j, \sigma_{t}, x_{t}\right)}{\partial x^{2}} d x_{t} d x_{t}+\frac{1}{2} \frac{\partial^{2} h\left(t, j, \sigma_{t}, x_{t}\right)}{\partial \sigma^{2}} d \sigma_{t} d \sigma_{t}+\frac{1}{2} \frac{\partial^{2} h\left(t, j, \sigma_{t}, x_{t}\right)}{\partial \sigma \partial x} d x_{t} d \sigma_{t}
\end{aligned}
$$


From (52), we have:

$$
\begin{aligned}
d h\left(t, j, \sigma_{t}, x_{t}\right) & =\frac{\partial h\left(t, j, \sigma_{t}, x_{t}\right)}{\partial t} d t+\left(r(t, j)-\frac{1}{2} \sqrt{\sigma(t, j)}\right) \frac{\partial h\left(t, j, \sigma_{t}, x_{t}\right)}{\partial x} d t \\
& +a(t, j)\left(b(t, j)-\sigma(t, j) \frac{\partial h\left(t, j, \sigma_{t}, x_{t}\right)}{\partial \sigma} d t+\frac{1}{2} \sigma_{t} \frac{\partial^{2} h\left(t, j, \sigma_{t}, x_{t}\right)}{\partial x^{2}} d t\right. \\
& +\frac{1}{2} v^{2}(t, j) \sigma(t, j) \frac{\partial^{2} h\left(t, j, \sigma_{t}, x_{t}\right)}{\partial \sigma^{2}} d t+\frac{1}{2} v(t, j) \sigma(t, j) \frac{\partial^{2} h\left(t, j, \sigma_{t}, x_{t}\right)}{\partial \sigma \partial x} d t \\
& +\sqrt{\sigma(t, j)} \frac{\partial h\left(t, j \sigma_{t}, x_{t}\right)}{\partial x} d W_{t}^{1}+v(t, j) \sqrt{\sigma(t, j)} \frac{\partial h\left(t, j, \sigma_{t}, x_{t}\right)}{\partial \sigma} d W_{t}^{1} .
\end{aligned}
$$

As $h$ is defined through the means of conditional expectations, it is easy to prove that $h$ is therefore a martingale process with respect to $\left(\mathbb{P},\left(\mathbb{F}_{t}\right)_{t \in[0, T]}\right)$. Therefore, the bounded variation terms of the right hand side of equation (81) reduce to 0 .

$$
\begin{aligned}
& \frac{\partial h\left(t, j, \sigma_{t}, x_{t}\right)}{\partial t}+\left(r(t, j)-\frac{1}{2} \sqrt{\sigma(t, j)}\right) \frac{\partial h\left(t, j, \sigma_{t}, x_{t}\right)}{\partial x} \\
& +a(t, j)(b(t, j)-\sigma(t, j)) \frac{\partial h\left(t, j, \sigma_{t}, x_{t}\right)}{\partial \sigma}+\frac{1}{2} \sigma_{t} \frac{\partial^{2} h\left(t, j, \sigma_{t}, x_{t}\right)}{\partial x^{2}} \\
& +\frac{1}{2} v^{2}(t, j) \sigma(t, j) \frac{\partial^{2} h\left(t, j, \sigma_{t}, x_{t}\right)}{\partial \sigma^{2}}+\frac{1}{2} \rho v(t, j) \sigma(t, j) \frac{\partial^{2} h\left(t, j, \sigma_{t}, x_{t}\right)}{\partial \sigma \partial x} \\
& =0
\end{aligned}
$$

with boundary condition :

$$
h\left(u, j, y_{0}, \sigma_{0}, x_{0}\right)=e^{i w x_{0}} \text {. }
$$

From Elliott et al. (2015), we assume that the the characteristic function is of the form:

$$
h(t, j)=e^{\left(A_{t}+B_{t} \sigma_{t}+i w x_{t}\right)} .
$$

We apply substitution in (82) and from Elliott et al. (2015); Heston (1993) the following system of ODE is obtained:

$$
\begin{aligned}
& i r w+a(t, j) b(t, j) B_{t}+\dot{A}_{t}=0 \\
& -.5 w^{2}+i w \rho v B_{t}+.5 v^{2} B_{t}^{2}-.5 w-a B_{t}+\dot{B}_{t}=0
\end{aligned}
$$

Solutions of such a coupled system are found in Heston (1993) and Elliott et al. (2015).

$$
\begin{aligned}
B_{t} & =\frac{a-i \rho v w+\eta}{v^{2}}\left(\frac{1-e^{\eta(u-t)}}{1-\gamma e^{\eta(u-t)}}\right) \\
A_{t} & =i r w(u-t)+a \int_{t}^{u} B_{s} b_{s} d s \\
& \text { where, } \\
\eta & =\sqrt{(a-i \rho v w+\eta)^{2}+v^{2} w(w+i)} \text { and } \gamma=\frac{a-i \rho v w+\eta}{a-i \rho v w-\eta}
\end{aligned}
$$

We systematically extend the main result derived in Elliott et al. (2015) by considering semi Markov regimes. We find a similar but more general formula for vanilla call prices.

Lemma 3.4. Let $\boldsymbol{M}\left(t, y_{t}, u\right), u \geq t, \Theta_{t}, K$ and $\Lambda$ be an $m \times m$ real valued matrix function, a semi Markov process, the strike price of an option contract and the conditional intensity matrix of $\Theta_{t}$. We assume that $\boldsymbol{M}$ is solution of the matrix PDE:

$$
\frac{\partial \boldsymbol{M}}{\partial t}+\frac{\partial \boldsymbol{M}}{\partial y}+\boldsymbol{A}(t, y) \boldsymbol{M}(t, y, u)=0, \text { with } \boldsymbol{M}\left(u, y_{u}, u\right)=\boldsymbol{I} .
$$

1. The Vanilla European Heston call price from Carr and Madan algorithm is given by the semi analytic formula

$$
C\left(0, \Theta_{0}, y_{0}, x_{0}, v_{0}\right)=\frac{e^{-\alpha k}}{\pi} \operatorname{Re}\left(\int_{0}^{t} e^{i \phi k} \frac{e^{-r T} \psi(\phi-(1+\alpha))}{\alpha^{2}+\alpha-v^{2}+i(2 \alpha+1) \phi}\right),
$$


with $k=\log (K)$ and $\alpha$ the Carr and Madan parameter. $\psi$ is the characteristic function of the log asset price $x$, given by the following expression:

$$
\psi\left(u, \Theta_{0}, y_{0}, x_{0}, v_{0}, w\right)=E\left(e^{i \phi x_{u}} \mid \mathbb{F}_{0}\right)=e^{i r \phi u+B_{0} \sigma_{0}+i w x_{0}}\left\langle\Phi(u, 0, y) \Theta_{0}, \mathbf{1}\right\rangle,
$$

where $\Phi$ satisfies the equation (89) with $\boldsymbol{A}(t, y)=-\operatorname{diag}\left(\boldsymbol{f}\left(0, y_{0}\right)\right)+\Lambda^{T}\left(y_{0}\right)$ and with $f\left(t, y_{t}\right)=a B_{t} b\left(t, y_{t}\right)$.

2. A semi closed expression for vanilla option prices with Heston model in a market with semi Markov regimes is as follows:

$$
\begin{aligned}
C\left(u, \Theta_{0}, y_{0}, x_{0}, v_{0}\right)= & e^{-r u}\left(\frac{1}{2}+\frac{1}{\pi} \int_{0}^{\infty} \mathcal{R} e\left[\frac{e^{i w r u+i w x_{0}+B_{0} \sigma_{0}}\left\langle\Phi\left(u, 0, y_{0}\right) \Theta_{0}, \mathbf{1}\right\rangle}{i w}\right] d w\right) \\
& +K e^{-r u}\left(\frac{1}{2}+\frac{1}{\pi} \int_{0}^{\infty} \mathcal{R} e\left[\frac{e^{-i w \log (K)+i r w u+i b x_{0}+\bar{B}_{0} \sigma_{0}+i w x_{0}}\left\langle\bar{\Phi}\left(u, 0, y_{0}\right) \Theta_{0}, \mathbf{1}\right\rangle}{i w}\right] d w\right),
\end{aligned}
$$

where $\Phi$ (respectively, $\bar{\Phi}$ ) are solutions of the system of matrix partial differential equations (89) when $\boldsymbol{A}\left(t, y_{t}\right)=$ $-\operatorname{diag}\left(f\left(t, y_{t}\right)\right)+\Lambda^{T}\left(y_{t}\right)$ with $f\left(t, y_{t}\right)=a B_{t} b\left(t, y_{t}\right)$ (respectively, $f\left(t, y_{t}\right)=a \bar{B}_{t} b\left(t, y_{t}\right)$ ) with $A_{t}, B_{t}, \eta_{t}$ and $\gamma_{t}$ defined as in Lemma 3.3 for any $t>0$.

$$
\bar{B}_{t}=\frac{a-\rho v-i \rho v w+\bar{\eta}}{v^{2}}\left(\frac{1-e^{\bar{\eta}(u-t)}}{1-\bar{\gamma} e^{\bar{\eta}(u-t)}}\right),
$$

where,

$$
\begin{aligned}
& \bar{\eta}=\sqrt{(a-\rho v-i \rho v w+\bar{\eta})^{2}+v^{2} w(w+i)} \text { and } \bar{\gamma}=\frac{a-\rho v-i \rho v w+\bar{\eta}}{a-\rho v-i \rho v b-\bar{\eta}} \\
& \bar{A}_{t}=\operatorname{irw}(u-t)+a \int_{t}^{u} \bar{B}_{s} b_{s} d s .
\end{aligned}
$$

Proof. We note that (90) is a well known formula derived in Carr and Madan (1999). The critical issue is to prove (91). The first part of the lemma boils down to deriving an expression for the characteristic function of the log asset price of Heston model in a regime switching market.

$$
\begin{aligned}
E\left[e^{i w x_{t}} \mid \mathbb{F}_{0}\right] & =E\left[E\left[e^{i w x_{u}} \mid \mathbb{H}_{T} \vee \mathbb{L}_{0}\right] \mid \mathbb{F}_{0}\right] \\
& =e^{i w r u+i w x_{u}+B_{0} \sigma_{0}} E\left[e^{a \int_{0}^{u} B_{s} b\left(s, \theta_{s}^{-}, y_{s^{-}}\right) d s} \mid \mathbb{F}_{0}\right] \\
& =e^{i b r u+i b x_{0}+B_{0} \sigma_{0}}\left\langle\Phi\left(u, 0, y_{0}\right) \Theta_{0}, \mathbf{1}\right\rangle, \text { From Lemma 3.2, with } \Phi \text { satisfying (89) }
\end{aligned}
$$

where, $f\left(t, y_{t}\right)=a B_{t} b\left(t, y_{t}\right)$. This proves the first part of the lemma.

In order to complete the proof of the second part of the lemma, we recall the risk neutral pricing formula for a vanilla call option with initial $\operatorname{cost} C$, strike price $K$ and in the context of a regime switching Heston driven market:

$$
\begin{aligned}
C\left(u, \Theta_{0}, y_{0}, x_{0}, v_{0}\right) & =E\left[e^{-r u}\left(e^{x^{u}}-K\right)^{+} \mid \mathbb{F}_{0}\right] \\
& =e^{-r u} E\left[e^{x^{u}} 1_{x_{u} \geq \log K} \mid \mathbb{F}_{0}\right]-K e^{-r u} E\left[x_{u} \geq \log (K) \mid \mathbb{F}_{0}\right] \\
& =e^{-r u} E\left[e^{x^{u}} 1_{x_{u} \geq \log K} \mid \mathbb{F}_{0}\right]-K e^{-r u} \mathbb{P}\left(x_{u} \geq \log K \mid \mathbb{F}_{0}\right)
\end{aligned}
$$

The second term of the last equation has been expressed as a conditional survival probability. We will express the first term in similar fashion. We first define $\overline{\mathbb{P}}$ for any $A \in \mathbb{F}_{u}$ as follows:

$$
\begin{aligned}
\overline{\mathbb{P}}(A) & =\frac{1}{e^{x_{0}}} E\left[e^{-r u+x_{u}} 1_{A} \mid \mathbb{F}_{0}\right] \\
& =\frac{1}{e^{x_{0}}} E\left[e^{-.5 \int_{0}^{u} \sigma_{s} d s+\int_{0}^{u} \sqrt{\sigma_{s}} d B_{s}} 1_{A} \mid \mathbb{F}_{0}\right], \text { from }(52) .
\end{aligned}
$$

It is easy to prove via Itó formula that the process

$$
L_{t}=e^{-.5 \int_{0}^{u} \sigma_{s} d s+\int_{0}^{u} \sqrt{\sigma_{s}} d B_{s},}
$$


which satisfies the differential form of Itó lemma

$$
d L_{t}=L_{t} \int_{0}^{u} \sqrt{\sigma_{s}} d B_{s}
$$

is a martingale process Jacod and Shiryaev (1987) and consequently is an appropriate density process. Hence, from Girsanov theorem Applebaum (2009), $\overline{\mathbb{P}}$ is a probability measure absolutely continuous with respect to the conditional probability measure $\mathbb{P}\left(\mathbb{F}_{0}\right)$. The pricing formula can therefore be rewritten:

$$
C\left(u, \Theta_{0}, y_{0}, x_{0}, v_{0}\right)=e^{-r u} \overline{\mathbb{P}}\left(1_{x_{u} \geq \log K}\right)-K e^{-r u} \mathbb{P}\left(x_{u} \geq \log K \mid \mathbb{F}_{0}\right)
$$

We first derive an expression for the second survival probability of (95). Under the probability measure $\mathbb{P}$, the model follows the dynamic in (52). Hence,

$$
E\left[e^{i b x_{u}} \mid \mathbb{H}_{T} \vee \mathbb{L}_{0}\right]=e^{A_{0}+B_{0} \sigma_{0}+i b x_{0}}
$$

where,

$$
B_{t}=\frac{a-i \rho v b+\eta}{v^{2}}\left(\frac{1-e^{\eta(u-t)}}{1-\gamma e^{\eta(u-t)}}\right)
$$

where,

$$
\begin{aligned}
\eta & =\sqrt{(a-i \rho v b+\eta)^{2}+v^{2} b(b+i)} \text { and } \gamma=\frac{a-i \rho v b+\eta}{a-i \rho v b-\eta} \\
A_{t} & =\operatorname{irb}(u-t)+a \int_{t}^{u} B_{s} b_{s} d s
\end{aligned}
$$

therefore from Lemma 3.3, the characteristic function has the form,

$$
E\left[e^{i w x_{u}} \mid \mathbb{H}_{T} \vee \mathbb{L}_{0}\right]=e^{i w r u+i w x_{0}+B_{0} \sigma_{0}+a \int_{t}^{u} B_{s} b\left(s, \theta_{s}^{-}, y_{s}^{-}\right) d s}
$$

The only regime switching term involved is $b$. Hence,

$$
\begin{aligned}
E\left[e^{i w x_{t}} \mid \mathbb{F}_{0}\right] & =E\left[E\left[e^{i w x_{u}} \mid \mathbb{H}_{T} \vee \mathbb{L}_{0}\right] \mid \mathbb{F}_{0}\right] \\
& =e^{i w r u+i w x_{u}+B_{0} \sigma_{0}} E\left[e^{a \int_{0}^{u} B_{s} b\left(s, \theta_{s}^{-}, y_{s^{-}}\right) d s} \mid \mathbb{F}_{0}\right]
\end{aligned}
$$

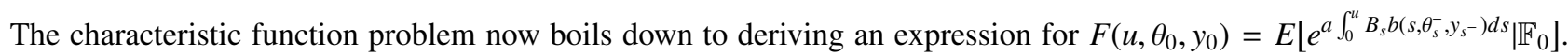
From Lemma (3.2) we have:

$$
\begin{aligned}
& F\left(u, \theta_{t}, y_{t}\right)=\left\langle\Phi\left(u, t, y_{t}\right) \Theta_{t}, \mathbf{1}\right\rangle, \text { with } \Phi \text { solution of the PDE: } \\
& \qquad \frac{\partial \Phi}{\partial t}+\frac{\partial \Phi}{\partial y}+\boldsymbol{A}\left(t, y_{t}\right) \Phi\left(u, t, y_{t}\right)=0, \text { with } \Phi\left(u, u, y_{u}\right)=\boldsymbol{I}, \forall u \geq t \geq 0
\end{aligned}
$$

where:

$$
\boldsymbol{A}\left(t, y_{t}\right)=\Lambda^{T}\left(y_{t}\right)-a B_{t} \operatorname{diag}\left(\boldsymbol{b}\left(t, y_{t}\right)\right) .
$$

Therefore the characteristic function and the survival probability sought are:

$$
\begin{aligned}
E\left[e^{i b x_{u}} \mid \mathbb{H}_{T} \vee \mathbb{L}_{0}\right] & =e^{i b r u+i b x_{0}+B_{0} \sigma_{0}} E\left[e^{a \int_{0}^{u} B_{s} b\left(s, \theta_{s}^{-}, y_{s}-\right) d s} \mid \mathbb{F}_{0}\right] \\
& =e^{i b r u+i b x_{0}+B_{0} \sigma_{0}}\left\langle\Phi\left(u, 0, y_{0}\right) \Theta_{0}, \mathbf{1}\right\rangle \\
\mathbb{P}\left(x_{u} \geq \log K \mid \mathbb{F}_{0}\right) & =\frac{1}{2}+\frac{1}{\pi} \int_{0}^{\infty} \mathcal{R}\left[\frac{e^{i b r u+i b x_{0}+B_{0} \sigma_{0}}\left\langle\Phi\left(u, 0, y_{0}\right) \Theta_{0}, \mathbf{1}\right\rangle}{i w}\right] d w .
\end{aligned}
$$

We turn our attention to deriving a semi closed expression for the first term of (95). We note that from Girsanov theorem Jacod and Shiryaev (1987), under the probability $\overline{\mathbb{P}}$, the standard Brownian motion in (52) become

$$
d \bar{B}_{t}=d B_{t}-\sqrt{\sigma_{t}} d t .
$$

The new dynamic of the Heston model under the probability measure $\overline{\mathbb{P}}$ is as follows:

$$
\begin{aligned}
d x_{t} & =\left(r+.5 \sigma_{t}\right) d t+\sqrt{\sigma_{t}} d \bar{B}_{t} \\
d \sigma_{t} & =\left(a_{t} b_{t}-\left(a_{t}-\rho v_{t}\right) \sigma_{t}\right)+v_{t} \sqrt{\sigma_{t}} d W_{t} .
\end{aligned}
$$


In a derivation similar to that of the first characteristic function and default probability, we obtain:

$$
\begin{aligned}
E\left[e^{i b x_{u}} \mid \mathbb{F}_{0}\right] & =e^{-i w \log (k)+i r w u+i b x_{0}+\bar{B}_{0} \sigma_{0}+i w x_{0}}\left\langle\bar{\Phi}\left(u, 0, y_{0}\right) \Theta_{0}, \mathbf{1}\right\rangle \\
\overline{\mathbb{P}}\left(x_{u} \geq \log K \mid \mathbb{F}_{0}\right) & =\frac{1}{2}+\frac{1}{\pi} \int_{0}^{\infty} \mathcal{R}\left[\frac{e^{-i w \log (k)+i r w u+i b x_{0}+\bar{B}_{0} v_{0}+i w x_{0}}\left\langle\bar{\Phi}\left(u, 0, y_{0}\right) \Theta_{0}, \mathbf{1}\right\rangle}{i w}\right] d w \\
& \frac{\partial \bar{\Phi}}{\partial t}+\frac{\partial \bar{\Phi}}{\partial y}+\boldsymbol{A}\left(t, y_{t}\right) \bar{\Phi}\left(u, t, y_{t}\right)=0, \text { with } \bar{\Phi}\left(u, u, y_{u}\right)=\boldsymbol{I} \\
& \text { where: } \\
\boldsymbol{A}\left(t, y_{t}\right) & =\Lambda^{T}\left(y_{t}\right)-a \bar{B}_{t} \operatorname{diag}\left(\boldsymbol{b}\left(t, y_{t}\right)\right), \\
\bar{B}_{t} & =\frac{a-\rho v-i \rho v w+\bar{\eta}}{v^{2}}\left(\frac{1-e^{\bar{\eta}(u-t)}}{\left.1-\bar{\gamma} e^{\bar{\eta}(u-t)}\right),}\right. \\
\text { where, } & \\
\bar{\eta} & =\sqrt{(a-\rho v-i \rho v w+\bar{\eta})^{2}+v^{2} w(w+i)} \text { and } \bar{\gamma}=\frac{a-\rho v-i \rho v w+\bar{\eta}}{a-\rho v-i \rho v b-\bar{\eta}} \\
\bar{A}_{t} & =i r w(u-t)+a \int_{t}^{u} \bar{B}_{s} b_{s} d s .
\end{aligned}
$$

Hence, (95) yields the result to be proved.

\section{Conclusion}

This paper aimed at calibrating the historical and the risk neutral parameters of two nonlinear coupled systems of semi Markov regime switching stochastic differential equations, respectively representing a basket of commodity prices and the risk neutral dynamic of a stock price. In the former case, we obtained closed form parameter estimates and in the latter case we obtained a couple of semi closed form formulas for European call option prices, hence proving the tractability of both models when the market follows a semi Markov dynamic.

\section{Acknowledgements}

This research was supported by the Mathematical Sciences Division, US Army Research Office by Grants No. W911NF12-1-0090 and W911NF-15-1-0182.

\section{References}

Andrew, P., \& Ronnie, S. (2014). A regime-switching heston model for vix and s\&p 500 implied volatilities.

David, A. (2009). Levy processes and stochastic calculus. Cambridge university press.

Fischer, B., \& Myron, S. (1973). The pricing of options and corporate liabilities.

Helyette, G., \& Yih, F. S. (2009). Modeling commodity prices under the cev model.

Jean, J., \& Albert, N. S. (1987). Limit theorems for stochastic processes, volume 288. Springer-Verlag Berlin.

Jin-Chuan, Duan. et al. (1995). The garch option pricing model.

John C. C., Jonathan, E. I., \& Stephen, A. R. (1985). A theory of the term structure of interest rates.

José, Da F., Martino, G., \& Claudio, T. (2008). A multifactor volatility heston model.

Olusegun, O., \& Ladde, G. (2014). Stochastic modeling of energy commoditys spot price processes with delay in volatility.

Olusegun, M. O., Gangaram, S. L., \& Nathan, G. L. (2016). Local lagged adapted generalized method of moments and applications.

Patrick, A., \& Ladde, S. G. (2015). Option pricing with a levy-type stochastic dynamic model for stock price process under semi-markovian structural perturbations. URL http://dx.doi.org/10.1142/S0219024915500521.

Patrick, A., \& Ladde, S. G. (2016). Simulation and calibration of options prices under a levy-type stochastic dynamic and semi markov market switching regimes processes. URL http://dx.doi.org/10.11114/aef.v4i1.1870.

Pierre, B. (1981). Point processes and queues, volume 30. Springer. Peter Carr \& Dilip Madan. (1999). Option valuation using the fast fourier transform. URL http://dx.doi.org/10.21314/JCF.1999.043.

Robert, C., \& Casella, G. (2013). Monte Carlo statistical methods. Springer Science \& Business Media. 
Robert, J. E., Katsumasa, N., \& Carlton, O. (2015). Heston-type stochastic volatility with a markov switching regime. https://doi.org/10.1002/fut.21761.

Steven, L. H. (1993). A closed-form solution for options with stochastic volatility with applications to bond and currency options.

\section{Copyrights}

Copyright for this article is retained by the author(s), with first publication rights granted to the journal.

This is an open-access article distributed under the terms and conditions of the Creative Commons Attribution license (http://creativecommons.org/licenses/by/4.0/). 